\title{
Nonlinear Flight Dynamics of Very Flexible Aircraft
}

\author{
Christopher M. Shearer * and Carlos E. S. Cesnik ${ }^{\dagger}$ \\ The University of Michigan, Ann Arbor, Michigan, 48109, USA
}

\begin{abstract}
This paper focuses on the characterization of the response of a very flexible aircraft in flight. The 6-DOF equations of motion of a reference point on the aircraft are coupled with the aeroelastic equations that govern the geometrically nonlinear structural response of the vehicle. A low-order strain-based nonlinear structural analysis coupled with unsteady finite-state potential flow aerodynamics form the basis for the aeroelastic model. The nonlinear beam structural model assumes constant strain over an element in extension, twist, and in/out of plane bending. The geometrically nonlinear structural formulation, the finite state aerodynamic model, and the nonlinear rigid body equations together provide a low-order complete nonlinear aircraft analysis tool. The equations of motion are integrated using an implicit modified generalized-alpha method. The method incorporates both first and second order nonlinear equations without the necessity of transforming the equations to first order and incorporates a Newton-Raphson sub-iteration scheme at each time step. Using the developed tool, analyses and simulations can be conducted which encompass nonlinear rigid body, nonlinear rigid body coupled with linearized structural solutions, and full nonlinear rigid body and structural solutions. Simulations are presented which highlight the importance of nonlinear structural modeling as compared to rigid body and linearized structural analyses in a representative High Altitude Long Endurance (HALE) vehicle. Results show significant differences in the three reference point axes (pitch, roll, and yaw) not previously captured by linearized or rigid body approaches. The simulations using both full and empty fuel states include level gliding descent, low-pass filtered square aileron input rolling/gliding descent, and low-pass square elevator input gliding descent. Results are compared for rigid body, linearized structural, and nonlinear structural response.
\end{abstract}

\section{Introduction}

Recent advances in airborne sensors and communication packages have brought the need for high-altitude long-endurance (HALE) aircraft. These platforms can be categorized under three broad missions, supporting either the military or civilian communities. The missions include airborne Intelligence, Surveillance, and Reconnaissance (ISR), for the military, ${ }^{1}$ network communication nodes for the military and civilian usage, ${ }^{2}$ and general atmospheric research. ${ }^{2}$ Due to the mission requirements, the desired vehicles are characterized by high-aspect-ratio wings, slender fuselages, and slender control surfaces, resulting in highly flexible vehicles. Examples of mission optimization studies for this class of vehicle can be found in Ref. 1, where the authors show HALE aircraft are required to have a fuel fraction greater than $66 \%$. This results in a very small structural weight fraction. The combination of high aerodynamic efficiency and low structural weight fraction yields inherently flexible wings. The HALE vehicle will then be susceptible to large dynamic wing deformations at low frequencies, presenting a direct impact into the flight dynamic characteristics of the vehicle.

Aircraft elastic flight dynamics have been studied and analyzed for more than three quarters of a century. However research and applications of flexible aircraft dynamics have been based primarily on linear models or, at best, nonlinear rigid body vehicle dynamics coupled with linear structural dynamics. For the majority of conventional aircraft, linear analysis has been very successful in providing sound aircraft designs. But when analyzing aircraft with high-aspect-ratio wings and large fuel fractions, linear aeroelastic analysis is shown to be ineffective in capturing the relevant aircraft dynamics. ${ }^{3}$

\footnotetext{
*Major, USAF, PhD Candidate, Student Member, shearerc@umich.edu.

$\dagger$ Associate Professor of Aerospace Engineering, AIAA Associate Fellow, cesnik@umich.edu.
} 


\section{A. Previous Work}

In the past decade, several researchers have investigated different aspects of flexible aircraft structural dynamics. Researchers have typically focused on various forms of the rigid body dynamics augmented with linear structural modeling. Newman and Buttrill ${ }^{4}$ utilized a linear aircraft model with linear structural modes and showed the difficulty in supressing aeroelastic dynamic effects from the rigid body response of a flexible supersonic transport. References 5, 6, and 7 have overcome the limitations of conventional control architecture design used in Ref. 4 by incorporating a dynamic inversion technique for large high speed flexible aircraft. While the dynamic inversion method is ideally suited for nonlinear models, Gregory augmented the aircraft equations of motion (EOM) with linear elastic models. Recently, Meirovitch and Tuzcu ${ }^{8}$ developed an integrated approach for analyzing the nonlinear rigid body dynamics coupled with a linear representation of the structure. Their approach addressed analytical dynamics, linear structural dynamics, aerodynamics, and controls. Additionally, several other researchers have used linearized structural dynamics in the modeling of flexible aircraft, e.g., Li and Agarwal, ${ }^{9}$ Nam et. al., ${ }^{10}$ Schmidt and Raney, ${ }^{11}$ and Chavez and Schmidt. ${ }^{12}$ While these approaches are applicable to a wide class of high performance flight vehicles, they are not sufficient to deal with the changing mass properties and low stiffness characteristics of very flexible aircraft.

Early work, which included the nonlinear effects of low stiffness aircraft, was conducted by van Schoor and von Flotow. ${ }^{13}$ Their work demonstrated the critical importance of including aircraft structural dynamics when analyzing aircraft flight dynamics of very flexible aircraft. They showed, using linearized analysis about nonlinear equilibrium points, a significant change in the classic "rigid" body modes when flexible structural modeling was included.

More recently, Patil et. al. ${ }^{3}$ showed a significant change in flight dynamic characteristics due to wing flexibility. Their work showed a significant difference between the short period and phugoid modes of a very flexible aircraft when comparing rigid body, linear aeroelastic, and nonlinear aeroelastic dynamics. The nonlinear dynamics were obtained by linearizing about a nonlinear equilibrium. In a parallel effort, Drela ${ }^{14}$ developed an integrated analysis tool for conceptual aerodynamic, structural, and control-law design of an aircraft. The method provides rapid analysis during the early phases of aircraft design.

Furthering the development of nonlinear structural analysis tools, Cesnik and Brown ${ }^{15,16}$ introduced the strain-based approach for the modeling of highly-flexible aircraft. In Ref. 16, HALE aircraft were modeled using a rigid fuselage and a highly flexible high-aspect-ratio wing. The nonlinear structural dynamic analysis is a strain-based approach solved in the time domain. Details of the formulation can be found in Refs. 15 and 16 .

All these studies have contributed in different ways toward the understanding of the nonlinear response and stability of highly flexible aircraft. However, due to its complex coupled nature, the problem is still far from being understood. For that one needs to fully couple the 6-DOF dynamics of the vehicle with its geometrically nonlinear aeroelasticity properties. In fact, the mishap of NASA's Helios aircraft ${ }^{17}$ has highlighted the importance of nonlinear analysis of very flexible aircraft. One of the key recommendations of the report was to "develop multidisciplinary (structures, aerodynamic, controls, etc) models, which can describe the nonlinear dynamic behavior of aircraft modifications or perform incremental flight-testing." Nonlinear analyses tools are highly desirable and are certainly the first step in the development of future highly flexible vehicles.

\section{B. Objective of the paper}

The objective of this paper is to present a coupled 6-DOF vehicle dynamics with a modified version of the nonlinear strain-based structural formulation ${ }^{16}$ for high-aspect-ratio lifting surfaces. The proposed formulation is used to analyze the differences between rigid body, linearized aeroelastic, and nonlinear aeroelastic aircraft dynamic responses in gliding, roll commanded, and pitch commanded flight. This formulation will be used in future as the basis for control design of highly flexible vehicles.

\section{Development of the Equations of Motion}

The primary goal in any analysis of aircraft flight dynamics is to understand the trajectory and orientation of a fixed body reference frame, $B$, at point $O$, which in general is not the aircraft's center of mass, Figure 1 . The means for propagating the reference frame, $B$, forward in time is done by deriving and integrating a 


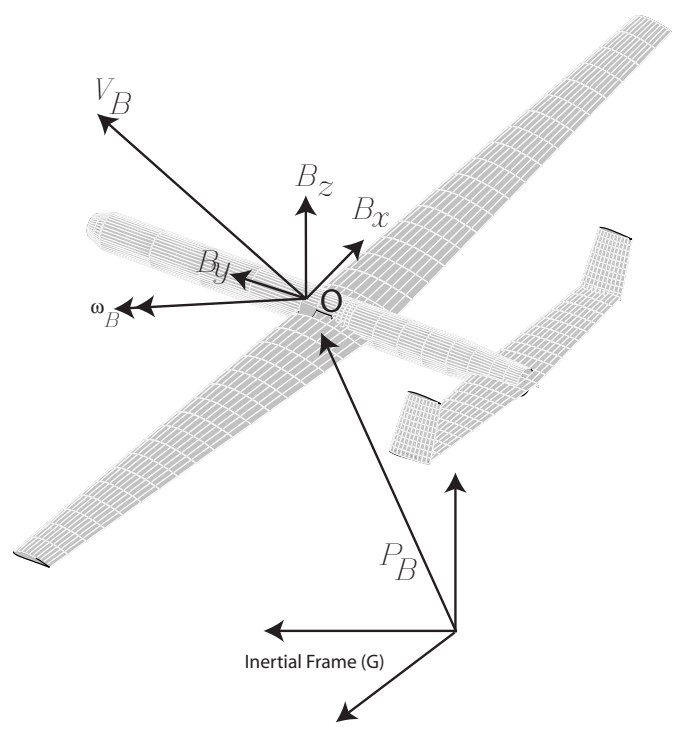

Figure 1. Basic Body Reference Frame and Vehicle Coordinates

series of first order differential equations of the form

$$
\dot{x}=f(x, u)
$$

where $x$ represents the states of the reference frame, $B$, and $u$ represents control surface and external inputs. Depending on the fidelity of the analysis required, these first order differential equations vary in their complexity from simple linear time invariant to non-linear time varying differential equations. For the classic "rigid" body analysis,${ }^{18}$ the first order differential equations take the form

$$
\begin{aligned}
\dot{v}_{B} & =f\left(v_{B}, \omega_{B}, \zeta, p_{B}, g_{0}^{\prime}, m, F\right) \\
\dot{\omega}_{B} & =f\left(\omega_{B}, I, \zeta, p_{B}, M\right) \\
\dot{\zeta} & =f(\zeta) \\
\dot{p}_{B} & =f\left(\zeta, v_{B}\right)
\end{aligned}
$$

where the $B$ reference frame linear and angular velocity variables are represented by $v_{B}$ and $\omega_{B} ; F$ and $M$ are in general state dependent external forces and moments; $m$ is the aircraft mass, and $I$ is the aircraft's inertia matrix. Where appropriate, these quantities are resolved in the $B$ coordinate frame. Additionally $\zeta$ is the vector of quaternion elements used to determine the orientation of the $B$ reference frame and $p_{B}$ is the inertial position of the $B$ reference frame. In this paper, three different representations of the $B$ reference frame are used: the nine components of the three unit vectors defining a triad in $B$ (used in the derivation of the flexible equations of motion), a minimal set of three rotation angles for transforming the flexible EOM to minimal set of states, and a set of four quaternion states for the time integration of the final set of EOM.

The rigid body formulation has three key assumptions which render invalid when dealing with very flexible vehicles: 1) inertia properties are constant or at best slowly time varying, 2) the inertial force associated with the vehicle's angular velocity cross with the relative velocity of flexible members is negligible, and 3) external forces and moments, $F$ and $M$, which are generated from aerodynamic loading, are based upon a fixed aircraft geometry. In the rigid body case, Eq. 2 presents only inertial and external forces and moments.

$$
3 \text { of } 26
$$


For the flexible aircraft a set of elastic EOM is also introduced, resulting in

$$
\begin{gathered}
M \ddot{q}+C \dot{q}+K q=R(q, \dot{q}, \lambda) \\
q=\left\{\begin{array}{c}
\epsilon \\
p_{B} \\
\Theta_{B}
\end{array}\right\} \quad \dot{q}=\left\{\begin{array}{c}
\dot{\epsilon} \\
v_{B} \\
\omega_{B}
\end{array}\right\} \quad \ddot{q}=\left\{\begin{array}{c}
\ddot{\epsilon} \\
\dot{v}_{B} \\
\dot{\omega}_{B}
\end{array}\right\}
\end{gathered}
$$

where $M$ represents generalized mass properties, $q$ is a set of generalized coordinates containing both strain, $\epsilon$, associated with the flexible vehicle and the inertial position, $p_{B}$, and orientation, $\Theta_{B}$, of the $B$ reference frame. $C$ contains both structural damping and nonlinear terms associated with relative position and velocity terms crossed with the absolute angular velocity, $K$ is the stiffness matrix, and $R(q, \dot{q}, \lambda)$ represents generalized forces (including aerodynamic forces) which are a function of the finite state inflow, $\lambda$. Coupling of the rigid body and flexible dynamics occurs through the dependency of $M, C$, and $R$. The present work uses a constant strain-based formulation ${ }^{16}$ which allows for airframe nonlinear geometric deformation and accounts for geometry-time-dependent inertia properties of the aircraft.

To develop the nonlinear elastic EOM for slender structures, a systematic approach is used based upon the principle of virtual work. The method accounts for the virtual work associated with rigid bodies and flexible slender (beam) structures. The virtual work of a beam is initially written in terms of dependent displacement vectors. Then the kinematic relationship between beam dependent position vectors and the associated strains is developed. The components of virtual work are summed and the resulting set of equations are transformed from a set of dependent position vectors and a nonminimum set of $B$ reference frame components to an independent set of strain variables, body linear and angular velocity and acceleration. Finally to account for the $B$ reference frame orientation and displacement a set of quaternions and inertial position differential equations are appended.

\section{A. Rigid Body Contribution to the Virtual Work}

To determine the rigid body contribution to the virtual work, the complete rigid body EOM are developed using a Lagrangian approach. The kinetic energy of a rigid body of mass, $m$, is

$$
\mathcal{T}=\frac{1}{2} \int_{m}\left(v_{p}^{B}\right)^{T} v_{p}^{B} d m
$$

where the inertial velocity, $v_{p}^{B}$, of an arbitrary point, $p$, expressed in the $B$ coordinate system is defined as

$$
v_{p}^{B}=v_{B}+\tilde{\omega}_{B} r_{c m}^{B}+\tilde{\omega}_{B} r_{p}^{B}
$$

where the position from the origin of the $B$ reference frame, $O$, to the center of mass $(\mathrm{cm})$ is $r_{c m}^{B}$, and $r_{p}^{B}$ is a vector of position coordinates from $\mathrm{cm}$ to $\mathrm{p}$. It is important to note that the $\mathrm{cm}$ described here is only for the rigid body portion of the flexible aircraft (which at the limit may be reduced to an arbitrary point in the vehicle). If the entire aircraft is modeled as a rigid body, than $\mathrm{cm}$ becomes the aircraft's center of mass. Throughout this paper the $(\tilde{\cdot})$ is the skew symmetric matrix such that, if $a \equiv\left[\begin{array}{lll}a_{1} & a_{2} & a_{3}\end{array}\right]^{T}$ then

$$
\tilde{a} \equiv\left[\begin{array}{ccc}
0 & -a_{3} & a_{2} \\
a_{3} & 0 & -a_{1} \\
-a_{2} & a_{1} & 0
\end{array}\right]
$$

and $\tilde{\tilde{r}})$ is the negative of $(\tilde{\cdot})$ such that

$$
\tilde{\tilde{a}} \equiv\left[\begin{array}{ccc}
0 & a_{3} & -a_{2} \\
-a_{3} & 0 & a_{1} \\
a_{2} & -a_{1} & 0
\end{array}\right]
$$

Expanding and integrating over the rigid body, the kinetic energy is

$$
\mathcal{T}=\frac{1}{2} m\left(v_{B}\right)^{T} v_{B}+\frac{1}{2}\left(\omega_{B}\right)^{T}\left[I-I_{r_{c m}}\right]\left(\omega_{B}\right)+\left(v_{B}\right)^{T} I_{m r}\left(\omega_{B}\right)
$$

$$
4 \text { of } 26
$$


where $I$ uses the standard definition of inertia matrices

$$
\begin{aligned}
I_{x x} & =\int_{m}\left(r_{p_{y}}^{2}+r_{p_{z}}^{2}\right) d m, \quad I_{x y}=-\int_{m} r_{p_{x}} r_{p_{y}} d m, \cdots \\
I & =\left[\begin{array}{lll}
I_{x x} & I_{x y} & I_{x z} \\
I_{x y} & I_{y y} & I_{y z} \\
I_{x z} & I_{y z} & I_{z z}
\end{array}\right]
\end{aligned}
$$

and $I_{r_{c m}}$ and $I_{m r}$ are

$$
\begin{aligned}
I_{r_{c m}} & =-m\left[\begin{array}{ccc}
r_{c m_{y}}^{2}+r_{c m_{z}}^{2} & -r_{c m_{x}} r_{c m_{y}} & -r_{c m_{x}} r_{c m_{z}} \\
-r_{c m_{x}} r_{c m_{y}} & r_{c m_{x}}^{2}+r_{c m_{z}}^{2} & -r_{c m_{y}} r_{c m_{z}} \\
-r_{c m_{x}} r_{c m_{z}} & -r_{c m_{y}} r_{c m_{z}} & r_{c m_{x}}^{2}+r_{c m_{y}}^{2}
\end{array}\right] \\
I_{m r} & =m\left[\begin{array}{ccc}
0 & r_{c m_{z}} & -r_{c m_{y}} \\
-r_{c m_{z}} & 0 & r_{c m_{x}} \\
r_{c m_{y}} & -r_{c m_{x}} & 0
\end{array}\right]
\end{aligned}
$$

The potential energy term, $V$, due to the gravity field, $g^{B}$, resolved in the body fixed coordinate frame is

$$
\begin{aligned}
\mathcal{V} & =\int_{m}\left(r_{o}^{B}+r_{c m}^{B}+r_{p}^{B}\right)^{T} g^{B} d m+\int_{m}\left\{\left(r_{c m}^{B}+{ }^{I} \tilde{\theta}^{B} r_{p}^{B}\right)\right\}^{T} g^{B} d m \\
& =m\left(r_{o}^{B}\right)^{T} g^{B}+m\left(r_{c m}^{B}\right)^{T} g^{B}+\left\{\int_{m}{ }^{I} \tilde{\theta}^{B} r_{c m}^{B} d m\right\}^{T} g^{B}
\end{aligned}
$$

Using Lagrange's equations yields

$$
M_{B} \ddot{\beta}+M_{B}\left[\begin{array}{cc}
\tilde{\omega}_{B} & 0 \\
0 & 0
\end{array}\right] \dot{\beta}+N_{B} g^{B}=R_{B}
$$

and the virtual work

$$
\delta W_{B}=\delta b\left(-M_{B} \dot{\beta}-M_{B}\left[\begin{array}{cc}
\tilde{\omega}_{B} & 0 \\
0 & 0
\end{array}\right] \beta-N_{B} g^{B}+R_{B}\right)
$$

where

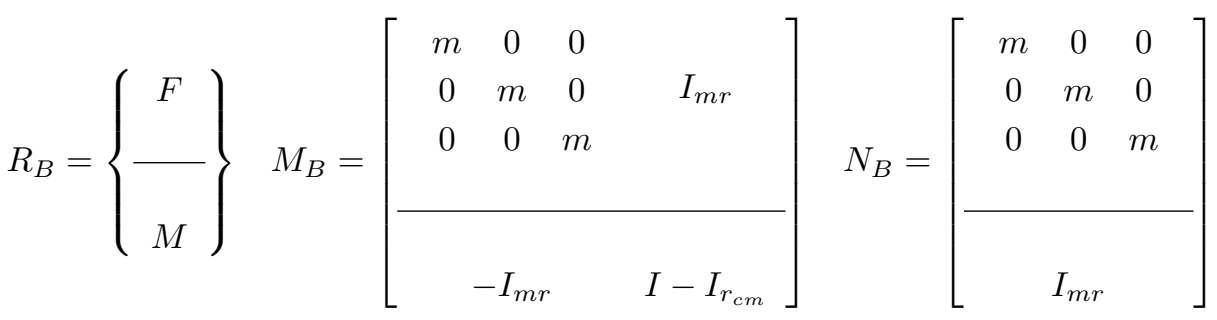

$$
\begin{aligned}
& b=\left\{\begin{array}{c}
p_{B} \\
\Theta_{B}
\end{array}\right\} \beta=\left\{\begin{array}{c}
v_{B} \\
- \\
\omega_{B}
\end{array}\right\} \quad \dot{\beta}=\left\{\begin{array}{c}
\dot{v}_{B} \\
\hline \\
\dot{\omega}_{B}
\end{array}\right\}
\end{aligned}
$$

\section{B. Flexible Slender Structure Contribution to the Virtual Work}

To develop the EOM, first define an arbitrary point in the body as

$$
p_{a}=p_{B}+p+x w_{x}+y w_{y}+z w_{z}
$$

$$
5 \text { of } 26
$$


where $p_{B}$ is the inertial position of the body fixed reference frame $B, p$ is the vector from the $B$ reference frame to a local reference frame $w$, and the constant scalars $x, y$, and $z$ are values along the corresponding $w$ frame orthogonal vectors $w_{x}, w_{y}$, and $w_{z}$. All the vectors are written in terms of the $B$ reference frame. The first and second time derivatives of $p_{a}$ can be written as

$$
\dot{p}_{a}=\dot{p}+x \dot{w}_{x}+y \dot{w}_{y}+z \dot{w}_{z}+\dot{p}_{B}+\tilde{\omega}_{B}\left(p+x w_{x}+y w_{y}+z w_{z}\right)
$$

and

$$
\begin{aligned}
\ddot{p}_{a}= & \ddot{p}_{B}+\dot{\tilde{\omega}}_{B}\left(p+x w_{x}+y w_{y}+z w_{z}\right)+\left(\ddot{p}+x \ddot{w}_{x}+y \ddot{w}_{y}+z \ddot{w}_{z}\right)+ \\
& +2 \tilde{\omega}_{B}\left(\dot{p}+x \dot{w}_{x}+y \dot{w}_{y}+z \dot{w}_{z}\right)+\tilde{\omega}_{B}\left(\dot{p}_{B}+\tilde{\omega}_{B}\left(p+x w_{x}+y w_{y}+z w_{z}\right)\right)
\end{aligned}
$$

Using the commutative property of the $(\tilde{\cdot})$ operator, Eq. 20 can be rewritten as

$$
\begin{aligned}
\ddot{p}_{a}= & \ddot{p}_{B}+\left(\tilde{\tilde{p}}+x \tilde{\tilde{w}}_{x}+y \tilde{\tilde{w}}_{y}+z \tilde{\tilde{w}}_{z}\right) \dot{\omega}_{B}+\left(\ddot{p}+x \ddot{w}_{x}+y \ddot{w}_{y}+z \ddot{w}_{z}\right)+ \\
& +2\left(\dot{\tilde{\tilde{p}}}+x \dot{\tilde{\tilde{w}}}_{x}+y \dot{\tilde{\tilde{w}}}_{y}+z \dot{\tilde{\tilde{w}}}_{z}\right) \omega_{B}+\tilde{\omega}_{B}\left(\dot{p}_{B}+\left(\tilde{\tilde{p}}+x \tilde{\tilde{w}}_{x}+y \tilde{\tilde{w}}_{y}+z \tilde{\tilde{w}}_{z}\right) \omega_{B}\right)
\end{aligned}
$$

Using d'Almbert's principle, the inertial force per unit volume is

$$
d F=-\rho \ddot{p}_{a} d A d s
$$

where $\rho$ is the density, $d A$ is the elemental area, and $d s$ is the elemental length. Then the virtual work per unit volume, $\delta W_{3}$, and its associated virtual displacement are

$$
\delta W_{3}=-\rho \delta p_{a}^{T} \ddot{p}_{a} \quad \delta p_{a}=\delta p+x \delta w_{x}+y \delta w_{y}+z \delta w_{z}
$$

where $\rho$ is considered to be prescribed over the elemental area and length. Using Eq. 21 in Eq. 23 yields

$$
\begin{aligned}
& \delta W_{3}=-\rho\left\{\delta p^{T}(1) \ddot{p}_{B}+\delta w_{x}^{T}(x) \ddot{p}_{B}+\delta w_{y}^{T}(y) \ddot{p}_{B}+\delta w_{z}^{T}(z) \ddot{p}_{B}+\right. \\
& +\left[\delta p^{T}(1) \tilde{\tilde{p}}+\delta w_{x}^{T}(x) \tilde{\tilde{p}}+\delta w_{y}^{T}(y) \tilde{\tilde{p}}+\delta w_{z}^{T}(z) \tilde{\tilde{p}}+\right. \\
& +\delta p^{T}(x) \tilde{\tilde{w}}_{x}+\delta w_{x}^{T}\left(x^{2}\right) \tilde{\tilde{w}}_{x}+\delta w_{y}^{T}(x y) \tilde{\tilde{w}}_{x}+\delta w_{z}^{T}(x z) \tilde{\tilde{w}}_{x}+ \\
& +\delta p^{T}(y) \tilde{\tilde{w}}_{y}+\delta w_{x}^{T}(x y) \tilde{\tilde{w}}_{y}+\delta w_{y}^{T}\left(y^{2}\right) \tilde{\tilde{w}}_{y}+\delta w_{z}^{T}(y z) \tilde{\tilde{w}}_{y}+ \\
& \left.+\delta p^{T}(z) \tilde{\tilde{w}}_{z}+\delta w_{x}^{T}(x z) \tilde{\tilde{w}}_{z}+\delta w_{y}^{T}(y z) \tilde{\tilde{w}}_{z}+\delta w_{z}^{T}\left(z^{2}\right) \tilde{\tilde{w}}_{z}\right] \dot{\omega}_{B}+ \\
& +\left[\delta p^{T}(1) \ddot{p}+\delta w_{x}^{T}(x) \ddot{p}+\delta w_{y}^{T}(y) \ddot{p}+\delta w_{z}^{T}(z) \ddot{p}+\right. \\
& +\delta p^{T}(x) \ddot{w}_{x}+\delta w_{x}^{T}\left(x^{2}\right) \ddot{w}_{x}+\delta w_{y}^{T}(x y) \ddot{w}_{x}+\delta w_{z}^{T}(x z) \ddot{w}_{x}+ \\
& +\delta p^{T}(y) \ddot{w}_{y}+\delta w_{x}^{T}(x y) \ddot{w}_{y}+\delta w_{y}^{T}\left(y^{2}\right) \ddot{w}_{y}+\delta w_{z}^{T}(y z) \ddot{w}_{y}+ \\
& \left.+\delta p^{T}(z) \ddot{w}_{z}+\delta w_{x}^{T}(x z) \ddot{w}_{z}+\delta w_{y}^{T}(y z) \ddot{w}_{z}+\delta w_{z}^{T}\left(z^{2}\right) \ddot{w}_{z}\right]+ \\
& +2\left[\delta p^{T}(1) \dot{\tilde{\tilde{p}}}+\delta w_{x}^{T}(x) \dot{\tilde{\tilde{p}}}+\delta w_{y}^{T}(y) \dot{\tilde{\tilde{p}}}+\delta w_{z}^{T}(z) \dot{\tilde{\tilde{p}}}+\right. \\
& +\delta p^{T}(x) \dot{\tilde{\tilde{w}}}_{x}+\delta w_{x}^{T}\left(x^{2}\right) \dot{\tilde{\tilde{w}}}_{x}+\delta w_{y}^{T}(x y) \dot{\tilde{\tilde{w}}}_{x}+\delta w_{z}^{T}(x z) \dot{\tilde{\tilde{w}}}_{x}+ \\
& +\delta p^{T}(y) \dot{\tilde{\tilde{w}}}_{y}+\delta w_{x}^{T}(x y) \dot{\tilde{\tilde{w}}}_{y}+\delta w_{y}^{T}\left(y^{2}\right) \dot{\tilde{\tilde{w}}}_{y}+\delta w_{z}^{T}(y z) \dot{\tilde{\tilde{w}}}_{y}+ \\
& \left.+\delta p^{T}(z) \dot{\tilde{\tilde{w}}}_{z}+\delta w_{x}^{T}(x z) \dot{\tilde{\tilde{w}}}_{z}+\delta w_{y}^{T}(y z) \dot{\tilde{\tilde{w}}}_{z}+\delta w_{z}^{T}\left(z^{2}\right) \dot{\tilde{\tilde{w}}}_{z}\right] \omega_{B}+ \\
& +\delta p^{T}(1)\left[\tilde{\omega}_{B}\right] \dot{p}_{B}+\delta w_{x}^{T}(x)\left[\tilde{\omega}_{B}\right] \dot{p}_{B}+\delta w_{y}^{T}(y)\left[\tilde{\omega}_{B}\right] \dot{p}_{B}+\delta w_{z}^{T}(z)\left[\tilde{\omega}_{B}\right] \dot{p}_{B}+ \\
& +\left[\delta p^{T}(1)\left[\tilde{\omega}_{B}\right] \tilde{\tilde{p}}+\delta w_{x}^{T}(x)\left[\tilde{\omega}_{B}\right] \tilde{\tilde{p}}+\delta w_{y}^{T}(y)\left[\tilde{\omega}_{B}\right] \tilde{\tilde{p}}+\delta w_{z}^{T}(z)\left[\tilde{\omega}_{B}\right] \tilde{\tilde{p}}+\right. \\
& +\delta p^{T}(x)\left[\tilde{\omega}_{B}\right] \tilde{\tilde{w}}_{x}+\delta w_{x}^{T}\left(x^{2}\right)\left[\tilde{\omega}_{B}\right] \tilde{\tilde{w}}_{x}+\delta w_{y}^{T}(x y)\left[\tilde{\omega}_{B}\right] \tilde{\tilde{w}}_{x}+\delta w_{z}^{T}(x z)\left[\tilde{\omega}_{B}\right] \tilde{\tilde{w}}_{x}+ \\
& +\delta p^{T}(y)\left[\tilde{\omega}_{B}\right] \tilde{\tilde{w}}_{y}+\delta w_{x}^{T}(x y)\left[\tilde{\omega}_{B}\right] \tilde{\tilde{w}}_{y}+\delta w_{y}^{T}\left(y^{2}\right)\left[\tilde{\omega}_{B}\right] \tilde{\tilde{w}}_{y}+\delta w_{z}^{T}(y z)\left[\tilde{\omega}_{B}\right] \tilde{\tilde{w}}_{y}+ \\
& \left.\left.+\delta p^{T}(z)\left[\tilde{\omega}_{B}\right] \tilde{\tilde{w}}_{z}+\delta w_{x}^{T}(x z)\left[\tilde{\omega}_{B}\right] \tilde{\tilde{w}}_{z}+\delta w_{y}^{T}(y z)\left[\tilde{\omega}_{B}\right] \tilde{\tilde{w}}_{z}+\delta w_{z}^{T}\left(z^{2}\right)\left[\tilde{\omega}_{B}\right] \tilde{\tilde{w}}_{z}\right] \omega_{B}\right\}
\end{aligned}
$$

$$
6 \text { of } 26
$$


Now since $\delta p^{T}, \tilde{\omega}_{B}$, and $\tilde{\tilde{(} \cdot)}$ are not functions of $d A$ or $d s$ the following cross sectional mass matrix is defined as

$$
M_{c s}=\int_{A} \rho\left[\begin{array}{cccc}
1 & x & y & z \\
x & x^{2} & x y & x z \\
y & x y & y^{2} & y z \\
z & x z & y z & z^{2}
\end{array}\right] d A
$$

so that the virtual work per unit length, $\delta W_{1}$, is written as

$$
\begin{aligned}
\delta W_{1}= & -\left\{\delta p_{a}^{T} M_{c s}\left[\begin{array}{cc}
I & \tilde{\tilde{p}} \\
0 & \tilde{\tilde{w}}_{x} \\
0 & \tilde{\tilde{w}}_{y} \\
0 & \tilde{\tilde{w}}_{z}
\end{array}\right] \dot{\beta}+\delta p_{a}^{T} M_{c s}\left[\begin{array}{c}
\ddot{p} \\
\ddot{w}_{x} \\
\ddot{w}_{y} \\
\ddot{w}_{z}
\end{array}\right]+2 \delta p_{a}^{T} M_{c s}\left[\begin{array}{cc}
0 & \dot{\tilde{\tilde{p}}} \\
0 & \dot{\tilde{\tilde{w}}}_{x} \\
0 & \dot{\tilde{\tilde{w}}}_{y} \\
0 & \dot{\tilde{\tilde{w}}}_{z}
\end{array}\right] \beta+\right. \\
& \left.\delta p_{a}^{T} M_{c s}\left[\begin{array}{cccc}
\tilde{\omega}_{B} & 0 & 0 & 0 \\
0 & \tilde{\omega}_{B} & 0 & 0 \\
0 & 0 & \tilde{\omega}_{B} & 0 \\
0 & 0 & 0 & \tilde{\omega}_{B}
\end{array}\right]\left[\begin{array}{cc}
I & \tilde{\tilde{p}} \\
0 & \tilde{\tilde{w}}_{x} \\
0 & \tilde{\tilde{w}}_{y} \\
0 & \tilde{\tilde{w}}_{z}
\end{array}\right] \beta\right\}
\end{aligned}
$$

Defining the vector $h$ as the position and orientation at a point in the flexible body,

$$
h=\left\{\begin{array}{llll}
p^{T} & w_{x}^{T} & w_{y}^{T} & w_{z}^{T}
\end{array}\right\}^{T}
$$

which is a vector function of only $\epsilon$ and $b$ (as shown below), then the total derivative and variation of $h$ with respect to the independent coordinates $\epsilon$ and $b$ are

$$
\delta h=J_{h \epsilon} \delta \epsilon+J_{h b} \delta b \quad d h=J_{h \epsilon} d \epsilon+J_{h b} d b
$$

where

$$
J_{h \epsilon} \equiv\left[\frac{\partial}{\partial \epsilon} h\right] \quad J_{h b} \equiv\left[\frac{\partial}{\partial b} h\right]
$$

and $\dot{h}$ is

$$
\dot{h}=J_{h \epsilon} \dot{\epsilon}+J_{h b} \dot{b}=J_{h \epsilon} \dot{\epsilon}+J_{h b} \beta
$$

The relative velocity of $h$ with respect to the $B$ reference frame is $J_{h \epsilon} \dot{\epsilon}$ and the velocity of $h$ due to the movement of the $B$ reference frame is $J_{h b} \beta$. The Jacobian, $J_{h b}$, is

$$
J_{h b}=\left[\begin{array}{cc}
I & \tilde{\tilde{p}} \\
0 & \tilde{\tilde{w}}_{x} \\
0 & \tilde{\tilde{w}}_{y} \\
0 & \tilde{\tilde{w}}_{z}
\end{array}\right]
$$

and $J_{h \epsilon}$ is provided in the Appendix. Now the relative acceleration due to $\epsilon$ can be written as

$$
\ddot{p}+\ddot{w}_{x}+\ddot{w}_{y}+\ddot{w}_{z}=\dot{J_{h \epsilon} \dot{\epsilon}}+J_{h \epsilon} \ddot{\epsilon}
$$

and

$$
\dot{J_{h \epsilon}}=\left[\frac{\partial}{\partial \epsilon} J_{h \epsilon}\right] \dot{\epsilon}
$$

Note that $\left[\frac{\partial}{\partial \epsilon} J_{h \epsilon}\right]$ is a three-dimensional matrix. Finally the virtual work per unit length is

$$
\begin{aligned}
& \delta W_{1}=\left[\begin{array}{ll}
\delta \epsilon^{T} & \delta b^{T}
\end{array}\right]\left(\left[\begin{array}{cc}
J_{h \epsilon}^{T} M_{c s} J_{h \epsilon} & J_{h \epsilon}^{T} M_{c s} J_{h b} \\
J_{h b}^{T} M_{c s} J_{h \epsilon} & J_{h b}^{T} M_{c s} J_{h b}
\end{array}\right]\left\{\begin{array}{c}
\ddot{\epsilon} \\
\dot{\beta}
\end{array}\right\}+\left[\begin{array}{cc}
J_{h \epsilon}^{T} M_{c s} \dot{J_{h \epsilon}} & J_{h \epsilon}^{T} M_{c s} H_{h b} \\
J_{h b}^{T} M_{c s} \dot{J_{h \epsilon}} & J_{h b}^{T} M_{c s} H_{h b}
\end{array}\right]\left\{\begin{array}{c}
\dot{\epsilon} \\
\beta
\end{array}\right\}+\right. \\
& \left.2\left[\begin{array}{cc}
0 & J_{h \epsilon}^{T} M_{c s} H_{h \dot{\epsilon} \dot{\beta}} \\
0 & J_{h b}^{T} M_{c s} H_{h \dot{\epsilon} \dot{\beta}}
\end{array}\right]\left\{\begin{array}{c}
\dot{\epsilon} \\
\beta
\end{array}\right\}+\left[\begin{array}{cc}
C_{c s} & 0 \\
0 & 0
\end{array}\right]\left\{\begin{array}{c}
\dot{\epsilon} \\
\beta
\end{array}\right\}+\left[\begin{array}{cc}
K_{c s} & 0 \\
0 & 0
\end{array}\right]\left\{\begin{array}{c}
\epsilon \\
b
\end{array}\right\}-R\right)
\end{aligned}
$$

$$
7 \text { of } 26
$$


where the generalized structural damping and stiffness matrices are $C_{c s}$ and $K_{c s}$ respectively. New definitions are introduced

$$
H_{h \dot{\epsilon} \dot{\beta}}=\left[\begin{array}{cc}
0 & \dot{\tilde{\tilde{p}}} \\
0 & \tilde{\tilde{\tilde{w}}}_{x} \\
0 & \tilde{\tilde{\tilde{w}}}_{y} \\
0 & \tilde{\tilde{\tilde{w}}}_{z}
\end{array}\right] ; \quad H_{h b}=\tilde{\Omega} J_{h b} ; \quad \tilde{\Omega}=\left[\begin{array}{cccc}
\tilde{\omega}_{B} & 0 & 0 & 0 \\
0 & \tilde{\omega}_{B} & 0 & 0 \\
0 & 0 & \tilde{\omega}_{B} & 0 \\
0 & 0 & 0 & \tilde{\omega}_{B}
\end{array}\right]
$$

Note the entries for $H_{h \dot{\epsilon} \dot{\beta}}$ come from the relative velocity of $h$

$$
J_{h \epsilon} \dot{\epsilon}=\left\{\begin{array}{cccccc}
\dot{p}_{x} & \dot{p}_{y} & \dot{p}_{z} & \dot{w}_{x x} & \cdots & \dot{w}_{z z}
\end{array}\right\}^{T}=\left\{\begin{array}{cccc}
\dot{p}^{T} & \dot{w}_{x}^{T} & \dot{w}_{y}^{T} & \dot{w}_{z}^{T}
\end{array}\right\}^{T}
$$

Before the virtual work per unit length is integrated to yield the total virtual work the kinematic relationship and subsequent discretization of $h$ with respect to $\epsilon, p_{B}$, and the $B$ reference frame is presented.

\section{Kinematic Relationship: Constant Strain Formulation}

The constant strain formulation developed by Cesnik and Brown ${ }^{15,16}$ is adopted and summarized below. To facilitate the solution of the EOM, a spacial finite element discretization of the flexible equations is introduced here. The basic assumption is that the strain vector within a discrete beam element

$$
\boldsymbol{\epsilon}(t)=\left\{\begin{array}{llll}
\epsilon_{x}(t) & \kappa_{x}(t) & \kappa_{y}(t) & \kappa_{z}(t)
\end{array}\right\}^{T}
$$

is spatially constant and time dependent. The strain vector is comprised of beam extension $\left(\epsilon_{x}(t)\right)$, twist $\left(\kappa_{x}(t)\right)$, and the two bending components $\left(\kappa_{y}(t)\right.$ and $\left.\kappa_{z}(t)\right)$. The gradient of the position vector $h$ along the one dimensional beam coordinate, $s$, is given by

$$
\frac{\partial}{\partial s} h(s)=\bar{K}_{\epsilon}(s) h(s)
$$

where

$$
\bar{K}_{\epsilon}(s)=\left[\begin{array}{cccc}
0 & \overline{\mathcal{E}}_{x} & 0 & 0 \\
0 & 0 & \bar{\kappa}_{z} & -\bar{\kappa}_{y} \\
0 & -\bar{\kappa}_{z} & 0 & \bar{\kappa}_{x} \\
0 & \bar{\kappa}_{y} & -\bar{\kappa}_{x} & 0
\end{array}\right]
$$

and individual elements of $\bar{K}_{\epsilon}$ are $3 \times 3$ diagonal matrices such that

$$
\begin{array}{rccc}
\overline{\mathcal{E}}_{x}=\left[\begin{array}{ccc}
1+\epsilon_{x}(s) & 0 & 0 \\
0 & 1+\epsilon_{x}(s) & 0 \\
0 & 0 & 1+\epsilon_{x}(s)
\end{array}\right] & \bar{\kappa}_{z}=\left[\begin{array}{ccc}
\kappa_{z}(s) & 0 & 0 \\
0 & \kappa_{z}(s) & 0 \\
0 & 0 & \kappa_{z}(s)
\end{array}\right] \\
\bar{\kappa}_{y}=\left[\begin{array}{ccc}
\kappa_{y}(s) & 0 & 0 \\
0 & \kappa_{y}(s) & 0 \\
0 & 0 & \kappa_{y}(s)
\end{array}\right] & \bar{\kappa}_{x}=\left[\begin{array}{ccc}
\kappa_{x}(s) & 0 & 0 \\
0 & \kappa_{x}(s) & 0 \\
0 & 0 & \kappa_{x}(s)
\end{array}\right]
\end{array}
$$

Since the strain vector is constant over an element, Eq. 38 is simply a spatially varying linear ordinary differential equation with constant coefficients with respect to $s$. Then, the relation between $h$ and $\epsilon$ can be stated as

$$
h(s)=e^{\left(s-s_{0}\right) * \bar{K}_{\epsilon}} h_{b c}
$$

where $h_{b c}$ is the boundary condition and $s_{0}$ is the starting location along an element. The individual components of an element position vector $h$ are the three position components of $\mathbf{p}_{w}$ and the 9 components of the element reference frame $w$, all expressed in the $B$ reference system, Figure 2 .

$$
h(s, t)=\left\lfloor\begin{array}{llllllllllll}
p_{x} & p_{y} & p_{z} & w_{x x} & w_{x y} & w_{x z} & w_{y x} & w_{y y} & w_{y z} & w_{z x} & w_{z y} & w_{z z}
\end{array}\right\rfloor^{T}
$$

$$
8 \text { of } 26
$$




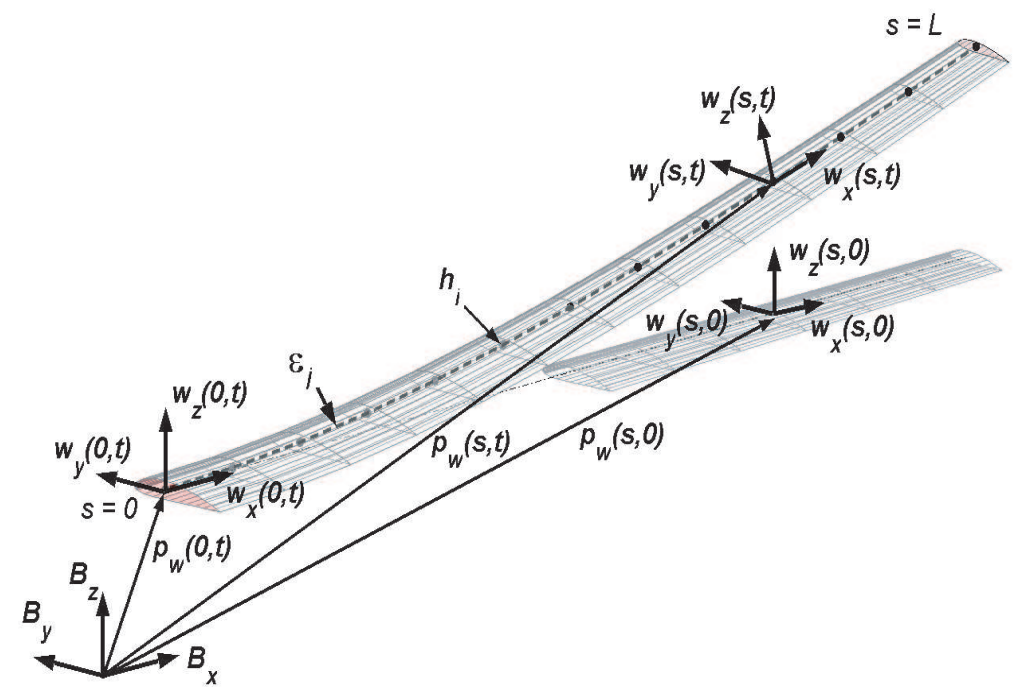

Figure 2. Geometry of Flexible Lifting Surface and Its Coordinate Systems

\section{Discrete Nodal Positions and the Position Vector $h$}

In the present formulation, each element, $i$, is comprised of three equally spaced nodes, with corresponding position vectors $h_{i, 1}, h_{i, 2}, h_{i, 3}$. Defining the unstrained geometric length of an element as $\Delta s=s-s_{0}$, and

$$
\bar{G}=\frac{\Delta s}{2} \bar{K}_{\epsilon}
$$

the kinematic relationship of the first three position vectors is

$$
h_{1,1}=h_{b c} \quad h_{1,2}=e^{\bar{G}} h_{b c} \quad h_{1,3}=e^{2 \bar{G}} h_{b c}
$$

A collection of elements, defined as a member (denoted by a superscript number), has the boundary condition defined as the position and orientation of the $B$ reference frame in Figure 1 or the position and orientation of an arbitrary element's $w$ reference frame. Here only members that start at point $O$ are discussed. Members are composed of an arbitrary number of key points and elements. In Figure 3, the key point 1 (KP1) is taken to have the boundary condition, $h_{b c}$.

$$
h_{b c}=\left\lfloor\begin{array}{llllllllllll}
p_{B_{x}} & p_{B_{y}} & p_{B_{z}} & B_{x x} & B_{x y} & B_{x z} & B_{y x} & B_{y y} & B_{y z} & B_{z x} & B_{z y} & B_{z z}
\end{array}\right\rfloor^{T}
$$

The column vector $h_{b c}$ contains the position and orientation components of the $B$ reference frame expressed in an inertial coordinate system, Figure 1. While Figure 3] shows a single element between each KP, the formulation allows a user defined number of elements between KPs. In general KPs are used to distinguish slope discontinuities in the beam reference line. Additionally, the beam reference line is allowed to have a linear variation in twist between KPs, but otherwise is a straight line when unstrained. Given the arrange-

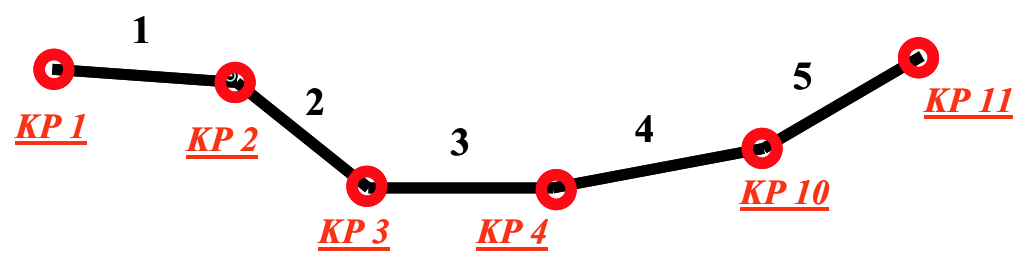

Figure 3. Member Discretization Showing Key Points (KP)

ment in Figure 3, the kinematic relationship between the element position and strain vectors for a member 


$$
\begin{aligned}
h_{1,1}^{1} & =h_{b c} \\
h_{1,2}^{1} & =e^{\bar{G}_{1}} h_{b c} \\
h_{1,3}^{1} & =e^{2 \bar{G}_{1}} h_{b c} \\
h_{2,1}^{1} & =D_{21} e^{2 \bar{G}_{1}} h_{b c} \\
h_{2,2}^{1} & =e^{\bar{G}_{2}} D_{21} e^{2 \bar{G}_{1}} h_{b c} \\
\vdots & \vdots \\
h_{5,3}^{1} & =e^{2 \bar{G}_{5}} D_{54} e^{2 \bar{G}_{4}} D_{43} e^{2 \bar{G}_{3}} D_{32} e^{2 \bar{G}_{2}} D_{21} e^{2 \bar{G}_{1}} h_{b c}
\end{aligned}
$$

where $D_{m, n}$ is a connecting matrix between elements $m$ and $n$ and accounts for slope discontinuities. For example, $D_{2,1}$ in this case is expressed as:

$$
D_{2,1}=\left[\begin{array}{cccc}
I & 0 & 0 & 0 \\
0 & c_{x x} * I & c_{y x} * I & c_{z x} * I \\
0 & c_{y x} * I & c_{y y} * I & c_{z y} * I \\
0 & c_{z x} * I & c_{y z} * I & c_{z z} * I
\end{array}\right]
$$

where $c_{i j}$ are the direction cosines between between element 1 and 2 .

\section{Closed Form Kinematic Relation}

Completing the derivation of the dependent position vector $h$ and the independent strain vector $\epsilon$, a closed form solution of $e^{\left(s-s_{0}\right) * \bar{K}_{\epsilon}}$ is proposed here. Using a transformation matrix, $T_{h}$, the original $h$ is reordered as $h^{*}$

$$
h^{*}=T_{h}^{T} h=\left\{\left(h_{x}^{*}\right)^{T} \quad\left(h_{y}^{*}\right)^{T} \quad\left(h_{z}^{*}\right)^{T}\right\}^{T}
$$

where

$$
h_{x}^{*}=\left\{\begin{array}{c}
p_{x}(s) \\
w_{x x}(s) \\
w_{y x}(s) \\
w_{z x}(s)
\end{array}\right\} \quad h_{y}^{*}=\left\{\begin{array}{c}
p_{y}(s) \\
w_{x y}(s) \\
w_{y y}(s) \\
w_{z y}(s)
\end{array}\right\} \quad h_{z}^{*}=\left\{\begin{array}{c}
p_{z}(s) \\
w_{x z}(s) \\
w_{y z}(s) \\
w_{z z}(s)
\end{array}\right\}
$$

The three corresponding differential equations are

$$
\frac{\partial}{\partial s} h_{x}^{*}=K_{\epsilon} h_{x}^{*} \quad \frac{\partial}{\partial s} h_{y}^{*}=K_{\epsilon} h_{y}^{*} \quad \frac{\partial}{\partial s} h_{z}^{*}=K_{\epsilon} h_{z}^{*}
$$

with

$$
K_{\epsilon}=\left[\begin{array}{cccc}
0 & \mathcal{E}_{x} & 0 & 0 \\
0 & 0 & \kappa_{z} & -\kappa_{y} \\
0 & -\kappa_{z} & 0 & \kappa_{x} \\
0 & \kappa_{y} & -\kappa_{x} & 0
\end{array}\right] \quad \mathcal{E}_{x} \equiv 1+\epsilon_{x}
$$

Using $T_{h}, \bar{K}_{\epsilon}$ is related to $\bar{K}_{\epsilon}^{*}$ by

$$
\bar{K}_{\epsilon}^{*}=T_{h}^{T} \bar{K}_{\epsilon} T_{h}=\left[\begin{array}{ccc}
K_{\epsilon} & 0 & 0 \\
0 & K_{\epsilon} & 0 \\
0 & 0 & K_{\epsilon}
\end{array}\right]
$$

and finally

$$
e^{\bar{K}_{\epsilon}^{*} *\left(s-s_{0}\right)}=\left[\begin{array}{ccc}
e^{K_{\epsilon} *\left(s-s_{0}\right)} & 0 & 0 \\
0 & e^{K_{\epsilon} *\left(s-s_{0}\right)} & 0 \\
0 & 0 & e^{K_{\epsilon} *\left(s-s_{0}\right)}
\end{array}\right]
$$

$$
10 \text { of } 26
$$


A closed form solution of the terms $e^{K_{\epsilon} *\left(s-s_{0}\right)}$ is found using the Cayley-Hamilton theorem, ${ }^{19}$ which allows any analytic matrix function to be expressed as a linear combination of the basis matrices

$$
f\left(K_{\epsilon}\right)=\alpha_{3}\left(K_{\epsilon}\right)^{3}+\alpha_{2}\left(K_{\epsilon}\right)^{2}+\alpha_{1} K_{\epsilon}+\alpha_{0} I
$$

To solve for the $\alpha_{i}$ coefficients, the eigenvalues of $K_{\epsilon}$ are found and substituted into the analytical expression for the function $f^{20}$

$$
\lambda_{1}=0 \quad \lambda_{2}=0 \quad \lambda_{3}=i \sqrt{\kappa_{x}^{2}+\kappa_{y}^{2}+\kappa_{z}^{2}} \quad \lambda_{4}=-i \sqrt{\kappa_{x}^{2}+\kappa_{y}^{2}+\kappa_{z}^{2}}
$$

Letting $\lambda=\sqrt{\kappa_{x}^{2}+\kappa_{y}^{2}+\kappa_{z}^{2}}$ and solving for $\alpha_{i}$

$$
\begin{gathered}
\alpha_{0}=1 \quad \alpha_{1}=s-s_{0} \\
\alpha_{2}=\frac{1-\cos \left(\lambda\left(s-s_{0}\right)\right)}{\lambda^{2}} \\
\alpha_{3}=\frac{\left(s-s_{0}\right)}{\lambda^{2}}-\frac{\sin \left(\lambda\left(s-s_{0}\right)\right)}{\lambda^{3}}
\end{gathered}
$$

yields the final closed form solution of $e^{K_{\epsilon} *\left(s-s_{0}\right)}$

$$
\begin{aligned}
e^{K_{\epsilon} *\left(s-s_{0}\right)}= & \left(\frac{\left(s-s_{0}\right)}{\lambda^{2}}-\frac{\sin \left(\lambda\left(s-s_{0}\right)\right)}{\lambda^{3}}\right)\left[\begin{array}{cccc}
0 & -\mathcal{E}_{x}\left(\kappa_{z}^{2}+\kappa_{y}^{2}\right) & \mathcal{E}_{x} \kappa_{y} \kappa_{x} & \mathcal{E}_{x} \kappa_{z} \kappa_{x} \\
0 & 0 & -\kappa_{z} \lambda^{2} & \kappa_{y} \lambda^{2} \\
0 & \kappa_{z} \lambda^{2} & 0 & -\kappa_{x} \lambda^{2} \\
0 & -\kappa_{y} \lambda^{2} & \kappa_{x} \lambda^{2} & 0
\end{array}\right]+ \\
& +\frac{1-\cos \left(\lambda\left(s-s_{0}\right)\right)}{\lambda^{2}}\left[\begin{array}{cccc}
0 & 0 & \mathcal{E}_{x} \kappa_{z} & -\mathcal{E}_{x} \kappa_{y} \\
0 & -\left(\lambda^{2}-\kappa_{x}^{2}\right) & \kappa_{y} \kappa_{x} & \kappa_{z} \kappa_{x} \\
0 & \kappa_{y} \kappa_{x} & -\left(\lambda^{2}-\kappa_{y}^{2}\right) & \kappa_{z} \kappa_{y} \\
0 & \kappa_{z} \kappa_{x} & \kappa_{z} \kappa_{y} & -\left(\lambda^{2}-\kappa_{z}^{2}\right)
\end{array}\right]+ \\
& +\left(s-s_{0}\right)\left[\begin{array}{ccccc}
0 & \mathcal{E}_{x} & 0 & 0 \\
0 & 0 & \kappa_{z} & -\kappa_{y} \\
0 & -\kappa_{z} & 0 & \kappa_{x} \\
0 & \kappa_{y} & -\kappa_{x} & 0
\end{array}\right]+\left[\begin{array}{cccc}
1 & 0 & 0 & 0 \\
0 & 1 & 0 & 0 \\
0 & 0 & 1 & 0 \\
0 & 0 & 0 & 1
\end{array}\right]
\end{aligned}
$$

which is used in Eqs. 41 and 52 .

\section{Element Discretization}

Using the assumption of a constant strain over an element, only the matrices $M_{c s}, C_{c s}$, and $K_{c s}$ are functions of the undeformed element length $s$. The current formulation uses a three-node element where the properties of the mass, structural damping, and stiffness are assumed to vary linearly between nodes of an element. Using this assumption an element mass matrix is written as

$$
M_{\text {element }}=\frac{1}{2} l\left[\begin{array}{ccc}
\frac{1}{4} M_{1}+\frac{1}{12} M_{2} & \frac{1}{12} M_{1}+\frac{1}{12} M_{2} & 0 \\
\frac{1}{12} M_{1}+\frac{1}{12} M_{2} & \frac{1}{12} M_{1}+\frac{1}{2} M_{2}+\frac{1}{12} M_{3} & \frac{1}{12} M_{2}+\frac{1}{12} M_{3} \\
0 & \frac{1}{12} M_{2}+\frac{1}{12} M_{3} & \frac{1}{12} M_{2}+\frac{1}{4} M_{3}
\end{array}\right]
$$

where $l$ is the length of the element and the subscripts 1,2 , and 3 are related to the lumped mass properties at each nodal position. In a similar manner, the element damping $C_{\text {element }}$ and stiffness $K_{\text {element }}$ matrices are found. For more details see Ref. 21.

\section{D. $B$ Reference Frame Propagation Differential Equations}

While the virtual work associated with the flexible EOM, Eq. 34, is derived using $\delta b$, the $B$ reference frame attitude propagation is accomplished using the first order differential quaternion equations described in

$$
11 \text { of } 26
$$


Ref. 18. The $B$ reference frame coordinate transformation matrix, $C^{B G}$, which transforms a vector from inertial coordinates to the $B$ frame coordinates, represented in terms of quaternions, $\zeta$, is given by

$$
C^{B G}=\left[\begin{array}{ccc}
B_{x} & B_{y} & B_{z}
\end{array}\right]=\left[\begin{array}{ccc}
\zeta_{0}^{2}+\zeta_{1}^{2}-\zeta_{2}^{2}-\zeta_{3}^{2} & 2\left(\zeta_{1} \zeta_{2}+\zeta_{0} \zeta_{3}\right) & 2\left(\zeta_{1} \zeta_{3}-\zeta_{0} \zeta_{2}\right) \\
2\left(\zeta_{1} \zeta_{2}-\zeta_{0} \zeta_{3}\right) & \zeta_{0}^{2}-\zeta_{1}^{2}+\zeta_{2}^{2}-\zeta_{3}^{2} & 2\left(\zeta_{2} \zeta_{3}+\zeta_{0} \zeta_{1}\right) \\
2\left(\zeta_{1} \zeta_{3}+\zeta_{0} \zeta_{2}\right) & 2\left(\zeta_{2} \zeta_{3}-\zeta_{0} \zeta_{1}\right) & \zeta_{0}^{2}-\zeta_{1}^{2}-\zeta_{2}^{2}+\zeta_{3}^{2}
\end{array}\right]
$$

where $B_{i}$ is shown in Figure 1, The first order differential equation governing the quaternion representation is given by ${ }^{18}$

$$
\left\{\begin{array}{c}
\dot{\zeta}_{0} \\
\dot{\zeta_{1}} \\
\dot{\zeta_{2}} \\
\dot{\zeta_{3}}
\end{array}\right\}=-\frac{1}{2}\left[\begin{array}{cccc}
0 & \omega_{B_{1}} & \omega_{B_{2}} & \omega_{B_{3}} \\
-\omega_{B_{1}} & 0 & -\omega_{B_{3}} & \omega_{B_{2}} \\
-\omega_{B_{2}} & \omega_{B_{3}} & 0 & -\omega_{B_{1}} \\
-\omega_{B_{3}} & -\omega_{B_{2}} & \omega_{B_{1}} & 0
\end{array}\right]\left\{\begin{array}{c}
\zeta_{0} \\
\zeta_{1} \\
\zeta_{2} \\
\zeta_{3}
\end{array}\right\} \equiv-\frac{1}{2} \Omega_{\zeta} \zeta
$$

where $\omega_{B_{i}}$ is the $i$-th component of body angular velocity. The inertial velocity of the $B$ reference frame is given by the differential equation

$$
\dot{p}_{B}=C^{B G} v_{B}
$$

\section{E. Unsteady Aerodynamics}

For this study, the unsteady aerodynamic forces and moments are calculated based upon the finite state aerodynamic theory of Peters and co-workers. ${ }^{22,23}$ The theory is derived for a two dimensional thin airfoil operating in inviscid and incompressible flow. The lift, $L^{\text {aero }}$, and the moment, $M^{\text {aero }}$, about the beam reference line excluding contributions from trailing edge control surfaces are given by

$$
\begin{aligned}
L^{\text {aero }} & =2 \pi \rho b\left(-\dot{y} \dot{z}+(b-d) \dot{y} \dot{\alpha}-\dot{y} \lambda_{0}-\frac{1}{2} b \ddot{z}-\frac{1}{2} b d \ddot{\alpha}\right) \\
M^{\text {aero }} & =d L^{\text {aero }}+2 \pi \rho b^{2}\left(-\frac{1}{2} \dot{y} \dot{z}-\frac{1}{2} d \dot{y} \dot{\alpha}-\frac{1}{2} \dot{y} \lambda_{0}-\frac{1}{16} b^{2} \ddot{\alpha}\right)
\end{aligned}
$$

where $b$ is the semi chord length and $d$ is the distance from the mid chord to the beam reference line. The velocity vector components are $\dot{y}$ along the chord and $\dot{z}$ perpendicular to the chord, and $\alpha$ is the angle of attack. The velocity components and angle of attack are derived from the beam nodal $h$ vector. The inflow velocity $\lambda_{0}$ is given by

$$
\lambda_{0}=\frac{1}{2} \sum_{n=1}^{N} b_{n} \lambda_{n}
$$

where the inflow states, $\lambda_{n}$, are governed by the differential equation

$$
\dot{\lambda}=E_{1} \lambda+E_{2} \ddot{z}+E_{3} \ddot{\alpha}+E_{4} \dot{\alpha}
$$

The matrices $E_{i}$ are given described in Ref. 22. The $b_{n}$ coefficients are binomial expansion coefficients given in appendix C, Ref. 23. While $\lambda_{0}$ is actually an infinite sum, $N=\infty, \lambda_{0}$ can be approximated with reasonable results by letting $N$ be between 4 and 8 .

To use this two dimensional theory for a three dimensional wing, a spanwise lift distribution function is used to correct each sectional lift and moment contribution.

$$
f_{L_{\text {corr }}}=1-e^{-\tau s}
$$

where $\tau$ is a user defined input that controls the spanwise lift deficiency correction. In the final form the generalized force and inflow equations take the form

$$
F^{a e r o}=f\left(q, \dot{q}, \lambda_{0}\right)
$$

$$
12 \text { of } 26
$$


and

$$
\dot{\lambda}=F_{1} \ddot{q}+F_{2} \dot{q}+F_{3} \lambda
$$

\section{F. Complete EOM}

Given the relationship between the dependent position vector $h$ and the independent vectors, $\epsilon$ and $b$, and summing up all the element virtual work contributions and the rigid body contributions, the total virtual work is written as

$$
\begin{aligned}
& \delta W=\left[\begin{array}{ll}
\delta \epsilon^{T} & \delta b^{T}
\end{array}\right]\left(-\left[\begin{array}{ll}
M_{F F} & M_{F B} \\
M_{B F} & M_{B B}
\end{array}\right]\left[\begin{array}{c}
\ddot{\epsilon} \\
\dot{\beta}
\end{array}\right]-\left[\begin{array}{ll}
C_{F F} & C_{F B} \\
C_{B F} & C_{B B}
\end{array}\right]\left[\begin{array}{c}
\dot{\epsilon} \\
\beta
\end{array}\right]+\right. \\
& \left.-\left[\begin{array}{ll}
K_{F F} & K_{F B} \\
K_{B F} & K_{B B}
\end{array}\right]\left[\begin{array}{c}
\epsilon \\
b
\end{array}\right]+R\right)
\end{aligned}
$$

where

$$
\begin{array}{ll}
M_{F F}=J_{h \epsilon}^{T} M J_{h \epsilon} & M_{F B}=J_{h \epsilon}^{T} M J_{h b} \\
M_{B F}=J_{h b}^{T} M J_{h \epsilon} & M_{B B}=J_{h b}^{T} M J_{h b}+M_{B} \\
C_{F F}=J_{h \epsilon}^{T} M \dot{J_{h \epsilon}}+C & C_{F B}=J_{h \epsilon}^{T} M H_{h b}+2 J_{h \epsilon}^{T} M H_{h \dot{\epsilon} \dot{\beta}} \\
C_{B F}=J_{h b}^{T} M \dot{J_{h \epsilon}} & C_{B B}=J_{h b}^{T} M H_{h b}+2 J_{h b}^{T} M H_{h \dot{\epsilon} \dot{\beta}}+M_{B}\left[\begin{array}{cc}
\tilde{\omega}_{B} & 0 \\
0 & 0
\end{array}\right] \\
K_{F F}=K & K_{F B}=0 \\
K_{B F}=0 & K_{B B}=0
\end{array}
$$

and $M, C$, and $K$ are the assembled flexible-element mass, damping, and stiffness matrices, respectively. The matrix $M_{B}$ represents the mass matrix associated with the rigid body portion of the structure. The resultant force vector $R$ is

$$
\begin{aligned}
R=\left\{\begin{array}{l}
R_{F} \\
R_{B}
\end{array}\right\}= & {\left[\begin{array}{l}
K_{F F} \\
K_{B F}
\end{array}\right] \epsilon_{\text {initial }}+\left[\begin{array}{l}
B_{g F} \\
B_{g B}
\end{array}\right] g^{B}+\left[\begin{array}{l}
B_{f d s t F} \\
B_{f d s t B}
\end{array}\right] F^{d s t}+\left[\begin{array}{c}
B_{M d s t F} \\
B_{M d s t B}
\end{array}\right] M^{d s t}+} \\
& +\left[\begin{array}{c}
B_{f p t F} \\
B_{f p t B}
\end{array}\right] F^{p t}+\left[\begin{array}{l}
B_{M p t F} \\
B_{M p t B}
\end{array}\right] M^{p t}
\end{aligned}
$$

where $\epsilon_{\text {initial }}$ is an initial strain vector, $g^{B}$ is the body-frame- $B$ resolved gravity vector, and $F^{d s t}, M^{d s t}, F^{p t}$, and $M^{p t}$, are body resolved distributed and point forces and moments. The aerodynamic forces and moments, $F^{a e r o}$ and $M^{\text {aero }}$, which are functions of control surface inputs, $u$, are included in $F^{d s t}$ and $M^{d s t}$. The remaining component details of the resultant force vector, $R$, are given in Ref. 16. From the Principle of Virtual Work, Eq. 67 yields

$$
\left[\begin{array}{ll}
M_{F F} & M_{F B} \\
M_{B F} & M_{B B}
\end{array}\right]\left[\begin{array}{c}
\ddot{\epsilon} \\
\dot{\beta}
\end{array}\right]+\left[\begin{array}{ll}
C_{F F} & C_{F B} \\
C_{B F} & C_{B B}
\end{array}\right]\left[\begin{array}{c}
\dot{\epsilon} \\
\beta
\end{array}\right]+\left[\begin{array}{ll}
K_{F F} & K_{F B} \\
K_{B F} & K_{B B}
\end{array}\right]\left[\begin{array}{l}
\epsilon \\
b
\end{array}\right]=R
$$

This set of equations can also be written in the compact form

$$
M_{C} \ddot{q}+C_{C} \dot{q}+K_{C} q=R(q, \dot{q}, \lambda)
$$

where the generalized mass and damping matrices are dependent on the strain state, while the generalized stiffness is constant. All the other nonlinearities are built in $R$.

The final governing differential equations are

$$
\begin{aligned}
M_{F F} \ddot{\epsilon} & =-M_{F B} \dot{\beta}-C_{F F} \dot{\epsilon}-C_{F B} \beta-K_{F F} \epsilon+R_{F} \\
M_{B B} \dot{\beta} & =-M_{B F} \ddot{\epsilon}-C_{B B} \beta-C_{B F} \dot{\epsilon}+R_{B} \\
\dot{\zeta} & =-\frac{1}{2} \Omega_{\zeta} \zeta \\
\dot{p}_{B} & =\left[\begin{array}{ll}
C^{B G} & 0
\end{array}\right] \beta \\
\dot{\lambda} & =F_{1} \ddot{q}+F_{2} \dot{q}+F_{3} \lambda
\end{aligned}
$$




\section{G. Solution of EOM}

To solve the nonlinear differential equations, Eq.71, a high frequency dissipative time stepping approach was implemented. The Generalized- $\alpha$ Method was selected based upon its ability to accurately integrate large systems of equations including ones with repeated eigenvalues. Moreover, the method also offers a relative ease of implementation with the current EOM modeling and the derivation of both a first and second order method. ${ }^{24,25}$ Both set of differential equations are integrated together and utilize a modification of Geradin' ${ }^{26}$ implicit integration method for time marching integration of nonlinear EOM.

The Generalized- $\alpha$ Method has the basic form ${ }^{25}$

$$
\begin{aligned}
\mathbf{d}_{n+1} & =\mathbf{d}_{n}+\Delta t \mathbf{v}_{n}+\Delta t^{2}\left(\left(\frac{1}{2}-\beta\right) \mathbf{a}_{n}+\beta \mathbf{a}_{n+1}\right) \\
\mathbf{v}_{n+1} & =\mathbf{v}_{n}+\Delta t\left((1-\gamma) \mathbf{a}_{n}+\gamma \mathbf{a}_{n+1}\right) \\
\mathbf{F}\left(t_{n+1-\alpha_{f}}\right) & =\mathbf{M} \mathbf{a}_{n+1-\alpha_{m}}+\mathbf{C} \mathbf{v}_{n+1-\alpha_{f}}+\mathbf{K} \mathbf{d}_{n+1-\alpha_{f}}
\end{aligned}
$$

where $\Delta t$ is the time step and

$$
\begin{aligned}
\mathbf{d}_{n+1-\alpha_{f}} & =\left(1-\alpha_{f}\right) \mathbf{d}_{n+1}+\alpha_{f} \mathbf{d}_{n} \\
\mathbf{v}_{n+1-\alpha_{f}} & =\left(1-\alpha_{f}\right) \mathbf{v}_{n+1}+\alpha_{f} \mathbf{v}_{n} \\
\mathbf{a}_{n+1-\alpha_{m}} & =\left(1-\alpha_{m}\right) \mathbf{a}_{n+1}+\alpha_{m} \mathbf{a}_{n} \\
t_{n+1-\alpha_{f}} & =\left(1-\alpha_{f}\right) t_{n+1}+\alpha_{f} t_{n}
\end{aligned}
$$

The parameters $\alpha_{f}, \alpha_{m}, \gamma$, and $\beta$ are used to control the amplification of high frequency numerical modes which are not of interest. For this application the following relationships were used

$$
\begin{aligned}
\gamma_{M C K} & =\frac{1}{2}-\alpha_{m}+\alpha_{f} \\
\beta_{M C K} & =\frac{1}{4}\left(1-\alpha_{m}+\alpha_{f}\right)^{2} \\
\alpha_{m_{M C K}} & =\frac{2 \rho_{\infty_{M C K}}-1}{\rho_{\infty_{M C K}}+1} \\
\alpha_{f_{M C K}} & =\frac{\rho_{\infty_{M C K}}}{\rho_{\infty_{M C K}}+1}
\end{aligned}
$$

where the subscript $M C K$ refers to the set of second-order differential EOM. The single parameter $\rho_{\infty}$ is used to control the numerical dissipation above the normalized frequency $\Delta t / T$, where $T$ is the period associated with the highest frequency of interest and

$$
0 \leq \rho_{\infty} \leq 1
$$

If $\rho_{\infty}$ is chosen to be unity than the trapezoidal method is obtained. If $\rho_{\infty}$ is chosen to be 0 than frequencies above $\Delta t / T$ will be dissipated in one time step, the so-called "asymptotic annihilation."

In a similar manner Jansen, Whiting and Hulbert ${ }^{24}$ developed the first order Generalized- $\alpha$ Method for a first order system of the form

$$
\dot{\lambda}=a \lambda
$$

where the following relationships were developed

$$
\begin{aligned}
\dot{\lambda}_{n+\alpha_{m}} & =a \lambda_{n+\alpha_{f}} \\
\lambda_{n+1} & =\lambda_{n}+\Delta t \dot{\lambda}_{n}+\Delta t \gamma\left(\dot{\lambda}_{n+1}-\dot{\lambda}_{n}\right) \\
\dot{\lambda}_{n+\alpha_{m}} & =\dot{\lambda}+\alpha_{m}\left(\dot{\lambda}_{n+1}-\dot{\lambda}_{n}\right) \\
\lambda_{n+\alpha_{f}} & =\lambda_{n}+\alpha_{f}\left(\lambda_{n+1}-\lambda_{n}\right)
\end{aligned}
$$

In a similar manner to the second order Generalized- $\alpha$ Method, the free parameters $\gamma, \alpha_{m}$, and $\alpha_{f}$ are chosen 


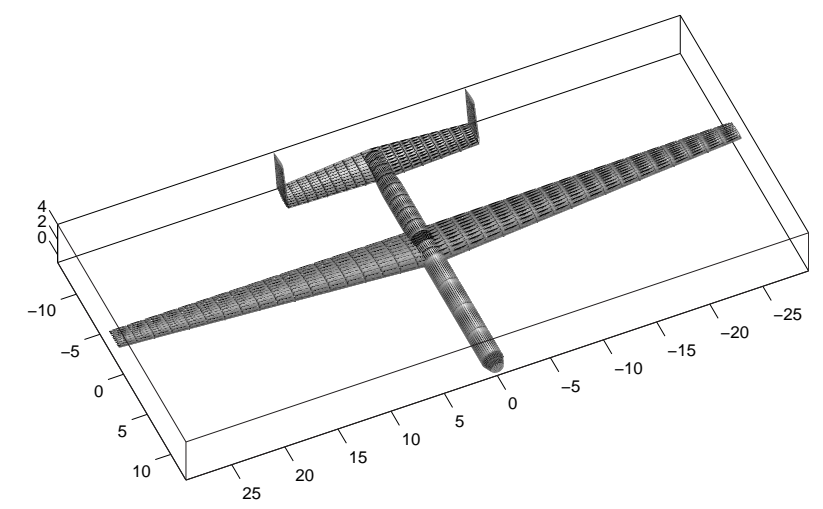

Figure 4. Twin Tailed Model - No Loading

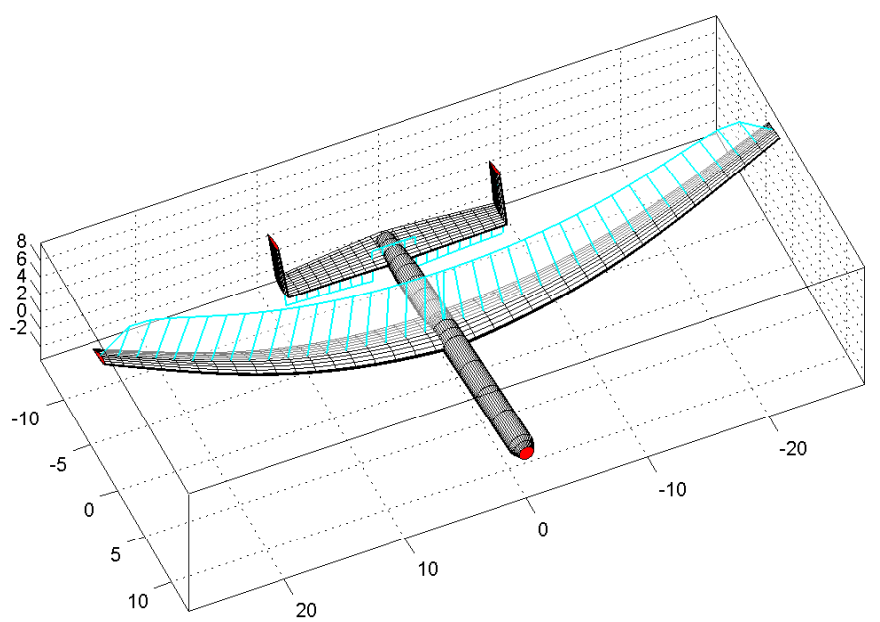

Figure 5. Flexible Aircraft Model at Steady State Deflection, Heavy Weight

in terms of a single high frequency spectral radius parameter $\rho_{\infty}$

$$
\begin{aligned}
\gamma_{\lambda} & =\frac{1}{2}+\alpha_{m_{\lambda}}-\alpha_{f_{\lambda}} \\
\alpha_{m_{\lambda}} & =\frac{1}{2}\left(\frac{3-\rho_{\infty_{\lambda}}}{1+\rho_{\infty_{\lambda}}}\right) \\
\alpha_{f_{\lambda}} & =\frac{1}{1+\rho_{\infty_{\lambda}}}
\end{aligned}
$$

\section{Numerical Example}

To exemplify the importance of the geometric nonlinear effects on the dynamic response of a typical HALE vehicle, a flexible aircraft model was developed as shown in Figure 4, It is a twin-tail conventional configuration with ailerons, rudders, and an elevator as control surfaces. The basic aircraft geometric and mass data are provided in Table 1 .

For each simulation case presented, three different solutions of Eq. 71 were simulated. The first is a reduced order solution where all elastic DOF are removed after the vehicle comes to a steady state deflection 
(rigid body with deformed structure and associated mass). A linearized solution retains the elastic DOF, but only uses the Jacobian matrices $\left(J_{h \epsilon}, J_{h b}\right.$, etc.) obtained from the steady state solution. The nonlinear solution starts from the steady state deflection, and updates the Jacobian matrices at each time step, i.e., a full time-marching simulation of Eq. 71 ,

\begin{tabular}{|c|c|c|c|}
\hline \multicolumn{4}{|c|}{ Model Parameters } \\
\hline \multirow[t]{2}{*}{ Property } & \multicolumn{2}{|c|}{ Value } & \multirow[t]{2}{*}{ Units } \\
\hline & Light & Heavy & \\
\hline Fuselage Length & \multicolumn{2}{|c|}{26.4} & $\mathrm{~m}$ \\
\hline Wing Span & \multicolumn{2}{|c|}{58.6} & $\mathrm{~m}$ \\
\hline Wing Area & \multicolumn{2}{|c|}{196.3} & $\mathrm{~m}^{2}$ \\
\hline Root Chord & \multicolumn{2}{|c|}{4.5} & $\mathrm{~m}$ \\
\hline Tip Chord & \multicolumn{2}{|c|}{2.2} & $\mathrm{~m}$ \\
\hline Aspect Ratio & \multicolumn{2}{|c|}{17.5} & - \\
\hline Horizontal Tail Span & \multicolumn{2}{|c|}{18.0} & $\mathrm{~m}$ \\
\hline Horizontal Root Chord & \multicolumn{2}{|c|}{3.5} & $\mathrm{~m}$ \\
\hline Horizontal Tip Chord & \multicolumn{2}{|c|}{2.45} & $\mathrm{~m}$ \\
\hline Vertical Tail Span & \multicolumn{2}{|c|}{4.0} & $\mathrm{~m}$ \\
\hline Vertical Root Chord & \multicolumn{2}{|c|}{2.45} & $\mathrm{~m}$ \\
\hline Vertical Tip Chord & \multicolumn{2}{|c|}{2.0} & $\mathrm{~m}$ \\
\hline Wing/Horizontal Tail Airfoil & \multicolumn{2}{|c|}{ NACA 4415} & - \\
\hline Vertical Tail Airfoil & \multicolumn{2}{|c|}{ NACA 0012} & - \\
\hline Aileron Location & \multicolumn{2}{|c|}{16.3 to 22.8} & $\mathrm{~m}$ \\
\hline Aileron, Elevator, Rudder Chord & \multicolumn{2}{|c|}{$0.2 c_{\text {local }}$} & \\
\hline Elevator Location & \multicolumn{2}{|c|}{1.8 to 9.0} & $\mathrm{~m}$ \\
\hline Rudder Location & \multicolumn{2}{|c|}{0.8 to 3.2} & $\mathrm{~m}$ \\
\hline Aircraft Angle of Attack & $0.64^{\circ}$ & $6.37^{\circ}$ & - \\
\hline Elevator Deflection Angle & $-4.11^{\circ}$ & $-13.43^{\circ}$ & - \\
\hline Fuel Mass & 0 & 32,000 & $\mathrm{~kg}$ \\
\hline Total Mass & $1.52 \cdot 10^{4}$ & $4.72 \cdot 10^{4}$ & $\mathrm{~kg}$ \\
\hline Fuel Fraction & 0.0 & 67.8 & $\%$ \\
\hline$I_{x x}^{s s *}$ & $9.61 \cdot 10^{5}$ & $1.17 \cdot 10^{6}$ & $\mathrm{~kg} \cdot \mathrm{m}^{2}$ \\
\hline$I_{y y}^{s s}$ & $8.21 \cdot 10^{5}$ & $2.94 \cdot 10^{6}$ & $\mathrm{~kg} \cdot \mathrm{m}^{2}$ \\
\hline$I_{z z}^{s s}$ & $1.75 \cdot 10^{6}$ & $3.93 \cdot 10^{6}$ & $\mathrm{~kg} \cdot \mathrm{m}^{2}$ \\
\hline$I_{x y}^{s s}$ & 0 & 0 & $\mathrm{~kg} \cdot \mathrm{m}^{2}$ \\
\hline$I_{x z}^{s s}$ & 0 & 0 & $\mathrm{~kg} \cdot \mathrm{m}^{2}$ \\
\hline$I_{y z}^{s s}$ & $-1.65 \cdot 10^{4}$ & $-4.72 \cdot 10^{4}$ & $\mathrm{~kg} \cdot \mathrm{m}^{2}$ \\
\hline Elements per wing & & & - \\
\hline Elements per horizontal tail & & & - \\
\hline Elements per vertical tail & & & - \\
\hline Elements in fuselage & & & - \\
\hline Total Number of Elements & & & - \\
\hline
\end{tabular}

*Note: $I^{s s}$ are the inertia properties in a steady state configuration

Table 1. Geometric and Inertia Properties of the Flexible Aircraft Model

Before any simulations were run the model was trimmed for zero pitching moment about the origin of the $B$ reference frame (which is the initial steady state center of mass of the aircraft) and 1-g level flight, 


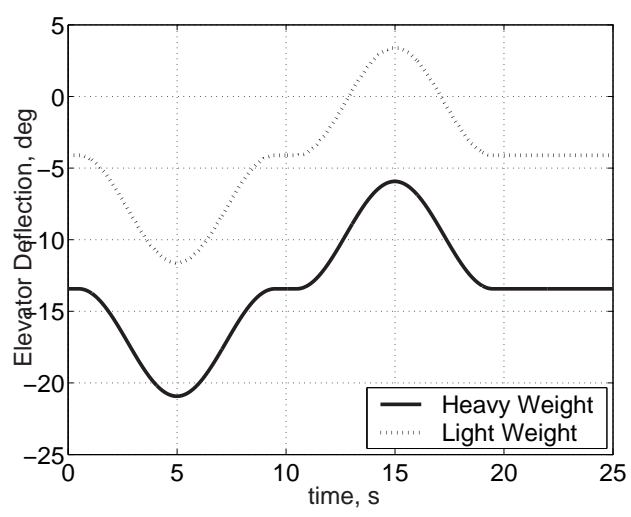

Figure 6. Elevator Input for Case 2 Heavy and Light Weights

shown in Figure 5 for heavy weight. Trimming for 1-g flight and zero pitching moment was accomplished by minimizing the following cost function

$$
J=f^{T} \cdot f
$$

where

$$
f=\left\{\begin{array}{c}
\text { pitching moment about the origin of } B \text { frame } \\
\text { lift }- \text { weight }
\end{array}\right\}
$$

The cost function $J$ was minimized over the solution space using the elevator deflection angle, $\delta_{e l}$, and the body angle of attack, $\alpha_{b o d}$. A simple numerical Newton-Raphson method was used to find the local minimum.

$$
\Delta u_{k}=-\left[\frac{\partial}{\partial u} f\right]_{k}^{-1} f_{k}
$$

where

$$
u=\left\{\begin{array}{c}
\delta_{e l} \\
\alpha_{b o d}
\end{array}\right\}
$$

Then $u$ is updated by

$$
u_{k+1}=u_{k}+\Delta u_{k}
$$

and $f_{k+1}$ and $\left[\frac{\partial}{\partial u} f\right]_{k+1}^{-1}$ are recomputed using $u_{k+1}$ and the process continues until the cost function $J$ reduces to some prescribed tolerance. To prevent divergence of the solution, $u_{k+1}$ was checked at each iteration step and kept within a prescribed set of bounds. The final values for $\delta_{e l}$ and $\alpha_{b o d}$ are given in Table 1 .

Three different cases were simulated at both heavy and light weight configurations. Case 1 is a simple straight-ahead descending flight. Case 2 is also descending flight, but a simple modified cosine elevator input was given to the aircraft as shown in Figure 6 to further excite the low elastic frequency modes. Case 3 is also a descending flight but with a modified cosine aileron input, as shown in Figure $7 \mathrm{~b}$, and a simple rudder command to alleviate the adverse yaw due to aileron input, as shown in Figure $7 \mathrm{c}$. The aileron input was chosen to achieve a maximum of approximately $45^{\circ}$ of bank using the reduced order solution. The rudder was determined using a heuristic approach to minimize the associated yaw rate. All three cases were simulated for 25 seconds with a constant time step of 0.01 seconds.

For Case 1, Figures 8 and 9 show the relevant body linear and angular velocities, $v_{B}$ and $\omega_{B}$. In both figures, the beginning of the classic phugoid mode can be observed, as indicated by the relatively large excursions in longitudinal velocity and small excursions in vertical velocity. For the heavy weight condition, the short period mode is seen as high frequency oscillations, as one can see in Figures $8 \mathrm{~b}$ and $8 \mathrm{c}$. As expected in traditional short period mode responses, the light weight configuration increases frequency and damping, hence smaller oscillations are observed in Figures $9 \mathrm{~b}$ and $9 \mathrm{k}$. In both the heavy and light weight conditions for this vehicle, little difference is seen between the linearized and nonlinear solutions for this case.

For case 2, similar results to Case 1 are shown in Figures 10 and 11, Larger variations in the body states are the main difference between Cases 1 and 2. This is due to the elevator input as defined in Figure [6. There

$$
17 \text { of } 26
$$




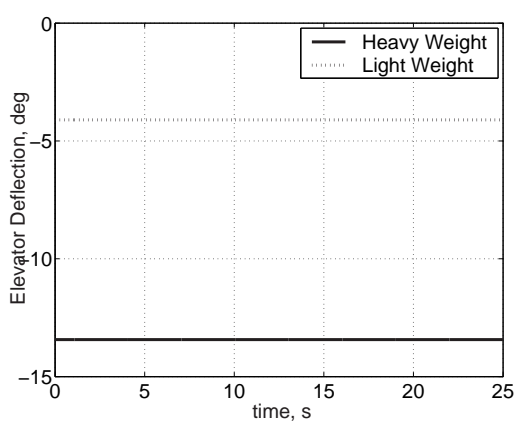

(a) Elevator

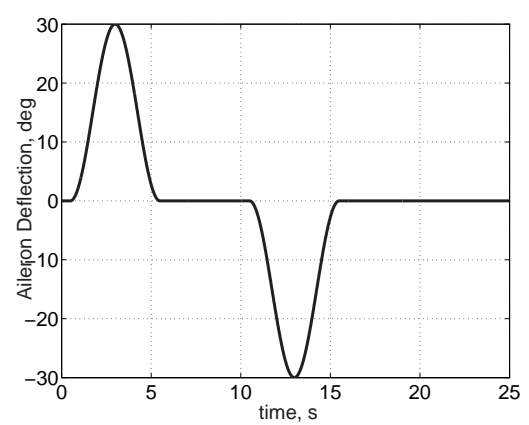

(b) Aileron

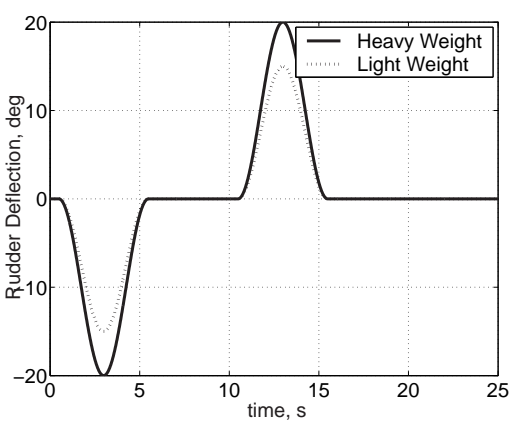

(c) Rudder

Figure 7. Control Inputs for Case 3, Heavy and Light Conditions

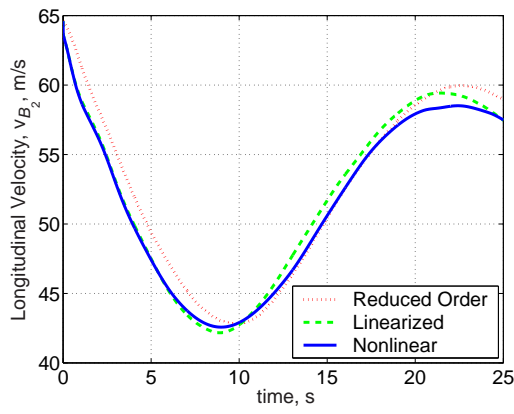

(a) Longitudinal Velocity

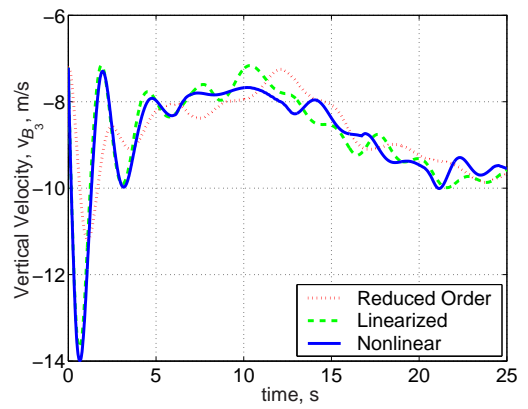

(b) Vertical Velocity

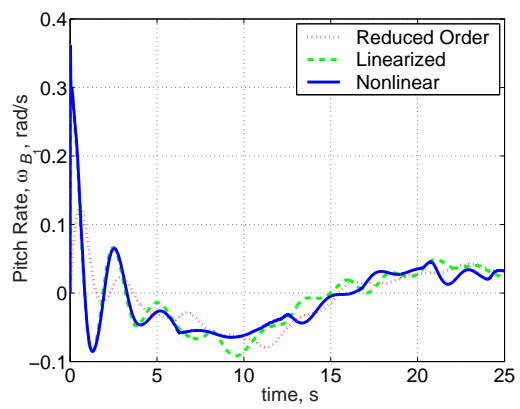

(c) Pitch Rate

Figure 8. $B$ Reference Frame Relevant Linear and Angular Rates, Case 1, Heavy

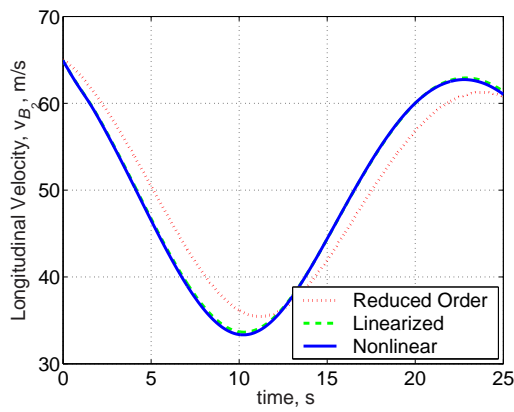

(a) Longitudinal Velocity

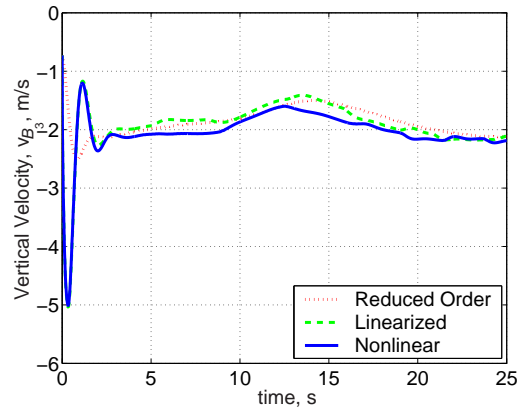

(b) Vertical Velocity

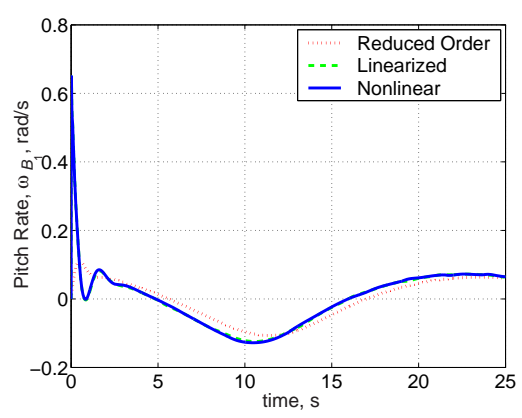

(c) Pitch Rate

Figure 9. $B$ Reference Frame Relevant Linear and Angular Rates, Case 1, Light 
are small variations between the linearized and nonlinear solutions, but essentially the linearized solution captures well the $B$ reference frame dynamics during symmetric loading and maneuvering. This is because the dominant changes associated with the generalized mass matrix (inertia about the longitudinal axis) do not couple with symmetric loading and maneuvering. This, however, is a very particular and restrictive case for an aircraft to perform any realistic mission. Therefore, asymmetric maneuvers must also be analyzed, as done next.

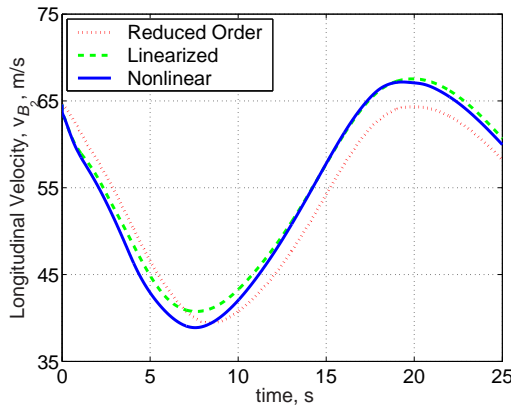

(a) Longitudinal Velocity

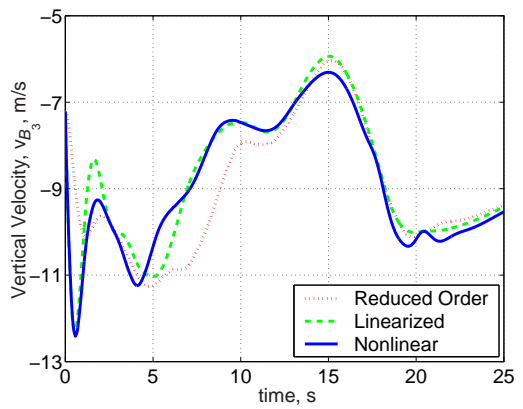

(b) Vertical Velocity

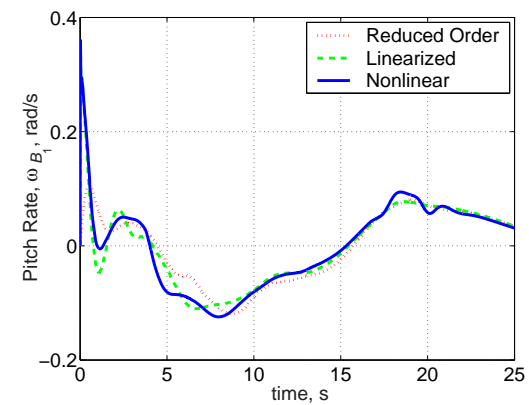

(c) Pitch Rate

Figure 10. $B$ Reference Frame Relevant Linear and Angular Rates, Case 2, Heavy

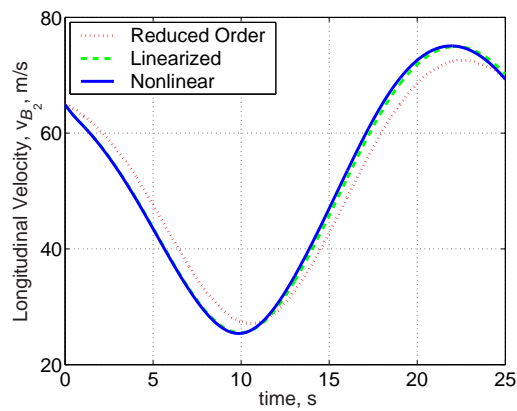

(a) Longitudinal Velocity

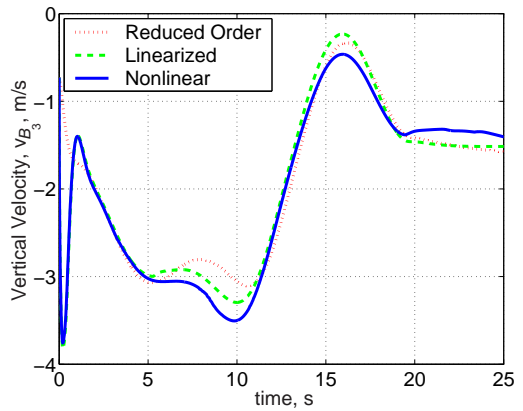

(b) Vertical Velocity

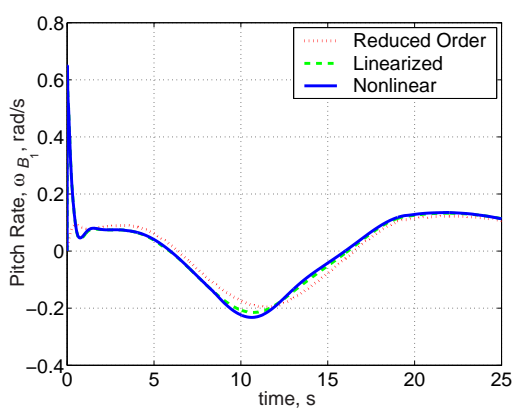

(c) Pitch Rate

Figure 11. B Reference Frame Relevant Linear and Angular Rates, Case 2, Light

Case 3 addresses the asymmetric loading (aileron and rudder input, as defined in Figure 7). Results for this case are shown in Figures 12 and 13. For the heavy weight condition, Figure 12, significant differences can be seen between the linearized and nonlinear solutions. For this case, the inertia effects tend to accentuate the geometrically nonlinear effects, driving the solution away from the linearized and reduced order solutions. Mathematically, the dominant changes in the generalized mass matrix do couple with the $B$ reference frame dynamics. However for a relatively high stiffness to mass ratio (a low fuel fraction-light weight), there is little difference between the nonlinear and linearized solutions, with the exception of the vertical velocity, as shown in Figure 130. In general, the reduced order approach performs poorly in predicting the linear and angular velocities of the example vehicle. To further highlight the differences in the orientation of the vehicle between the formulations, the classic Euler angles are recovered from the rotation matrix, ${ }^{18} C^{B G}$, and presented in Figure 14. At the end of the simulation interval $(t=25 \mathrm{~s})$, the linearized and nonlinear solutions disagree in the heading angle, $\Psi$, by almost $180^{\circ}$ (see Figure 14a) and in the bank angle, $\Phi$, by more than $40^{\circ}$ (see Figure 14 c).

In general, the results for Case 3 heavy weight show a significant divergence of the solutions from the three formulation approaches starting around 10 seconds. Looking closer to the vehicle flexible states at 15 seconds, one can see that the wings are under different vertical bending deflections, as illustrated in Figure 15. In fact, while the nonlinear formulation gives a wing tip deflection with respect to the initial trimmed condition of approximately $10 \%$ of the its semi-span, the linearized one gives $19 \%$. Clearly this is a deformation range that is outside the scope of linearized formulations. The difference is due to the geometric stiffening effect that is captured by the nonlinear formulation but not by the linearized one. To make matters 


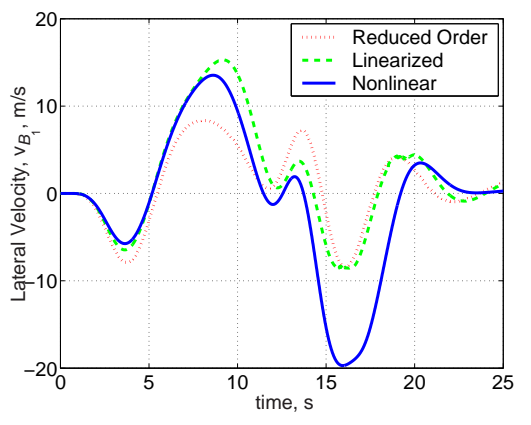

(a) Lateral Velocity

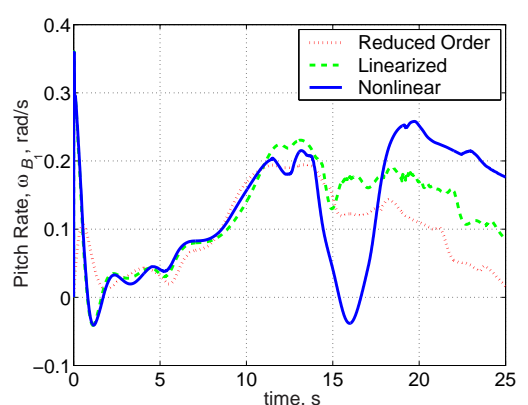

(d) Pitch Rate

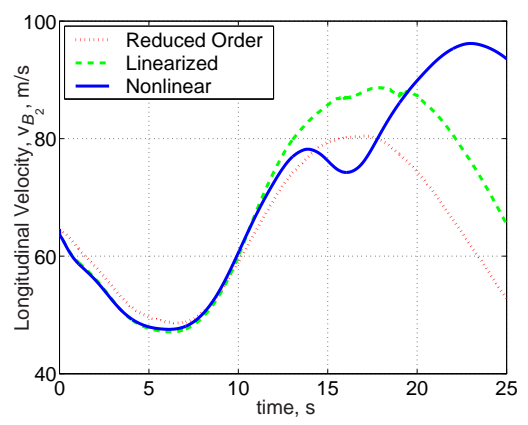

(b) Longitudinal Velocity

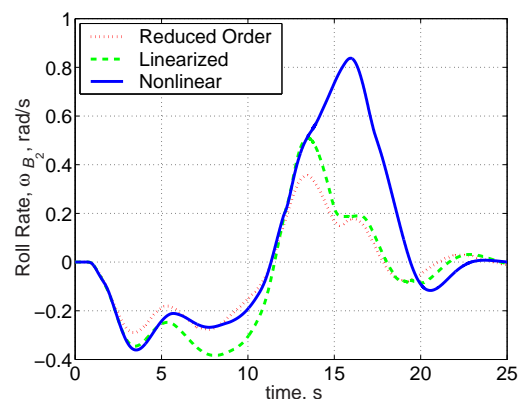

(e) Roll Rate

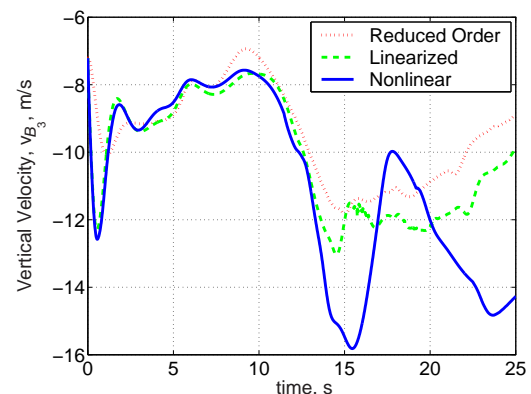

(c) Vertical Velocity

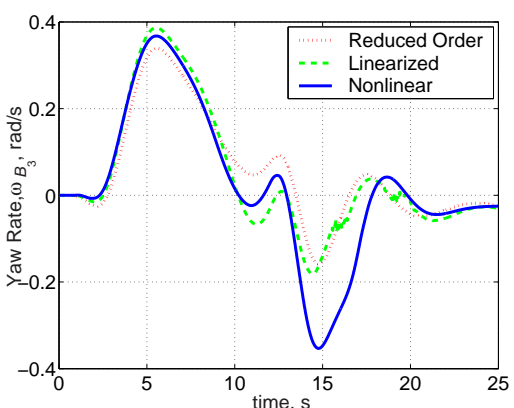

(f) Yaw Rate

Figure 12. $B$ Reference Frame Linear and Angular Rates, Case 3, Heavy

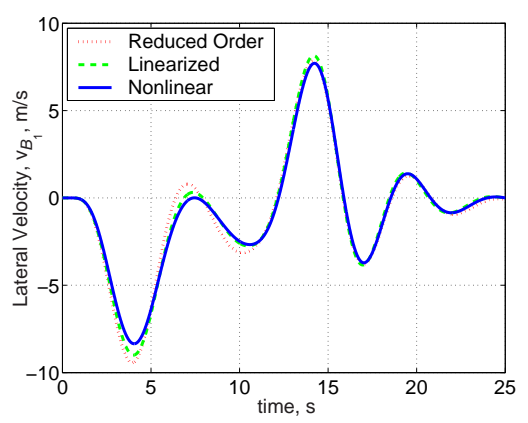

(a) Lateral Velocity

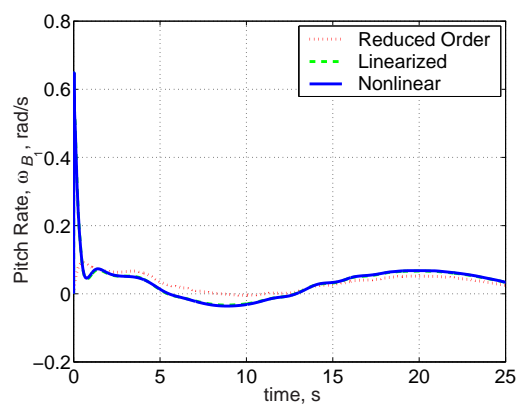

(d) Pitch Rate

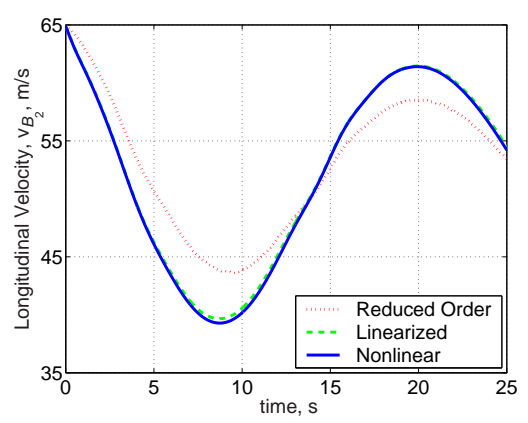

(b) Longitudinal Velocity

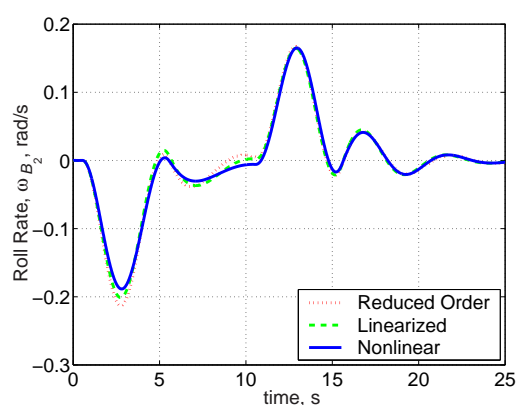

(e) Roll Rate

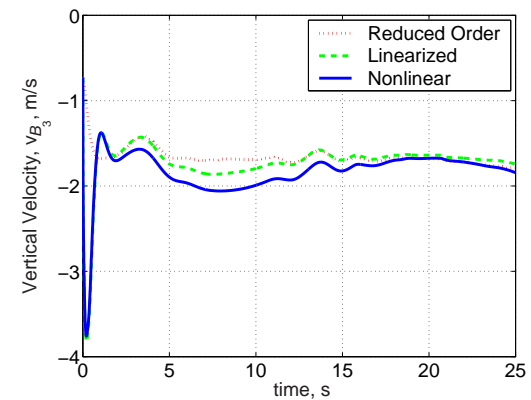

(c) Vertical Velocity

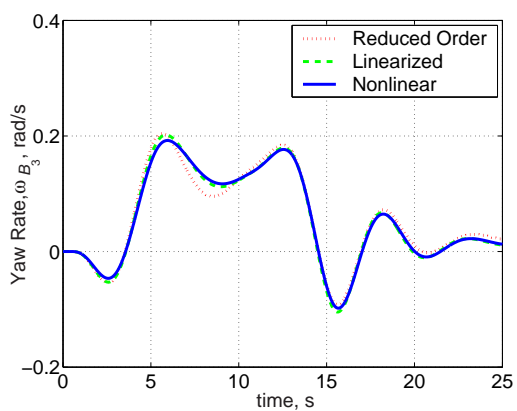

(f) Yaw Rate

Figure 13. $B$ Reference Frame Linear and Angular Rates, Case 3, Light 


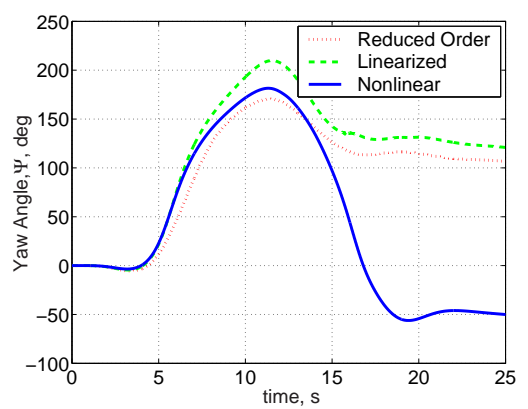

(a) Yaw Angle

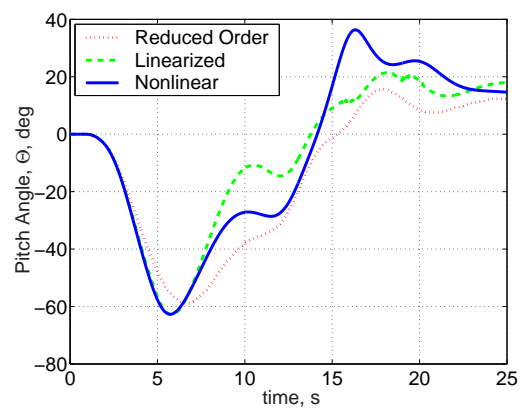

(b) Pitch Angle

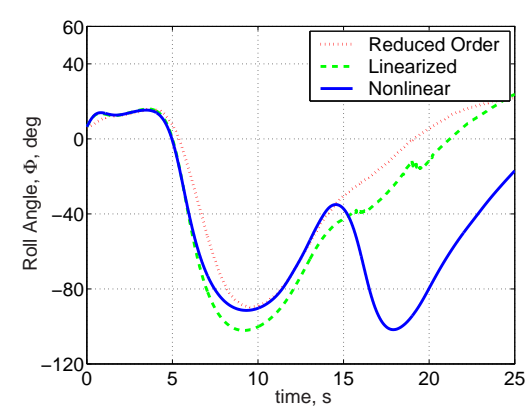

(c) Roll Angle

Figure 14. $B$ Reference Frame Classic Aircraft Euler Angles, Case 3, Heavy

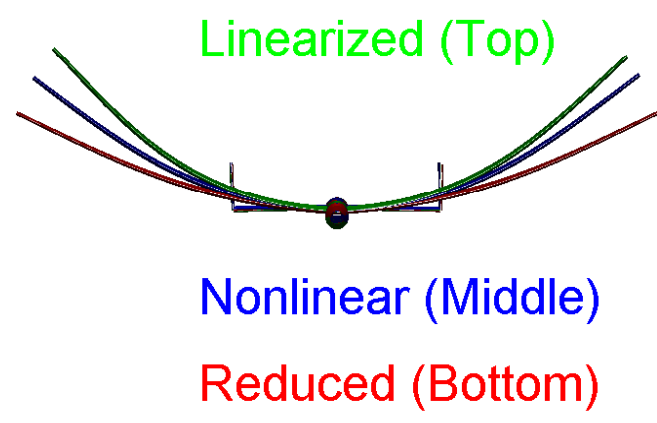

Figure 15. Head on View of Vehicle Deflection for Case 3 Heavy Weight, 15 seconds

more interesting, while the nonlinear formulation predicts a slightly higher left wing tip, the linearized one shows a higher right wing tip. As pointed out before, the reduced order approach is inherently flawed and does not capture the elastic motions of the vehicle that is altering its flight dynamics response.

\section{Concluding Remarks}

A framework for analyzing the flight dynamics of highly flexible vehicle configurations typically used in HALE aircraft was presented. It tighly couples the nonlinear 6-DOF equations of motion of a reference point in the aircraft with the aeroelastic equations that govern its geometrically nonlinear structural response. The structural dynamic analysis of the entire vehicle used a low-order strain-based geometrically nonlinear formulation. The unsteady aerodynamics used an incompressible finite-state potential flow formulation. The coupled nonlinear flight dynamics/aeroelastic equations of motion were then integrated using an implicit modified Generalized- $\alpha$ Method incorporating first and second order nonlinear differential equations. Using the proposed framework, analyses and simulations were conducted in a representative twin tailed HALE vehicle. The investigated cases compared nonlinear rigid body solutions, nonlinear vehicle 6-DOF coupled with tbe linearized aeroelastic solutions, and full nonlinear vehicle 6-DOF and aeroelastic solutions. Simulations 
used both full and empty fuel states for level gliding descent, low-pass square elevator input gliding descent, and low-pass filtered square aileron input rolling/gliding descent. In all heavy weight cases it was seen that the rigid body solutions were not sufficient to capture the dynamics of a very flexible aircraft and higher order formulations are required. When studying simple symmetric maneuvers, results indicated that linearized solutions may be acceptable to capture the main aircraft dynamics. However, when performing asymmetric maneuvering at heavy weight, results showed significant differences in the three reference point axes (pitch, roll, and yaw), requiring the nonlinear analysis approach to properly capture the vehicle response. Overall, simulation results showed the importance of having a minimum of a linearized structural analysis for symmetric maneuvering and nonlinear structural modeling for asymmetric maneuvering of a flexible aircraft like the ones being considered for high-altitude long-endurance civilian and military missions.

\section{Acknowledgments}

This work was partially sponsored by AFOSR grant F49620-02-1-0425. The technical monitor is Dr. Clark Allred, Capt. USAF. The views expressed in this article are those of the authors and do not reflect the official policy or position of the United States Air Force, Department of Defense, or the U.S. Government.

\section{References}

${ }^{1}$ Tilmann, C. P., Flick, P. M., Martin, C. A., and Love, M. H., "High-Altitude Long Endurance Technologies for SensorCraft," RTO AVT Symposium on "Novel Vehicle Concepts and Emerging Vehicle Technologies", Brussels, Belgium, April 7-10 2003, MP-104-P-26-1.

${ }^{2}$ Whitson, S., "The Proteus, Giving Shape to Forms Unknown," Private Pilot, Vol. 33, No. 12, December 1998, pp. 44-50.

${ }^{3}$ Patil, M. J., Hodges, D. H., and Cesnik, C. E. S., "Nonlinear Aeroelasticity and Flight Dynamics of High-Altitude LongEndurance Aircraft," 40th AIAA/ASME/ASCE/AHS/ASC Structures, Structural Dynamics, and Materials Conference and Exhibit, St. Louis, Missouri, April 12-15 1999, AIAA Paper No. 99-1470.

${ }^{4}$ Newman, B. and Buttrill, C., "Conventional Flight Control for an Aeroelastic Relaxed Static Stability High-Speed Transport," AIAA Guidance, Navigation and Control Conference, Baltimore, Maryland, August 7-10 1995, pp. 717-726, AIAA Paper No. 95-3250-CP.

${ }^{5}$ Gregory, I. M., "Dynamic Inversion to Control Large Flexible Transport Aircraft," AIAA Guidance, Navigation, and Control Conference and Exhibit, Boston, Massachusetts, August 10-12 1998, pp. 1224-1232, AIAA Paper No. 98-4323.

${ }^{6}$ Gregory, I. M., "Modified Dynamic Inversion to Control Large Flexible Transport Aircraft - What's Going On?" AIAA Guidance, Navigation, and Control Conference and Exhibit, Portland, Oregon, August 9-11 1999, pp. 392-402, AIAA Paper No. 99-3998.

${ }^{7}$ Gregory, I. M., "Stability Result for Dynamic Inversion Devised to Control Large Flexible Aircraft," AIAA Guidance, Navigation, and Control Conference and Exhibit, Montreal, Quebec, August 6-9 2001, AIAA Paper No. 2001-4284.

${ }^{8}$ Meirovitch, L. and Tuzcu, I., "Unified Theory for the Dynamics and Control of Maneuvering Flexible Aircraft," AIAA Journal, Vol. 42, No. 4, April 2004, pp. 714-727.

${ }^{9} \mathrm{Li}, \mathrm{X}$. and Agarwal, R. K., "Application of Reduced-Order-Models to Robust Control of the Dynamics of a Flexible Aircraft," AIAA Guidance, Navigation, and Control Conference and Exhibit, Austin, Texas, August 11-14 2003, AIAA Paper No. 2003-5504.

${ }^{10}$ Nam, C., Chen, P., Liu, D., Urnes, J., and Yurkovich, R., "Adaptive Reconfigurable Control Based on a Reduced Order System Identification for Flutter and Aeroservoelastic Instability Supression," 42nd AIAA/ASME/ASCE/AHS/ASC Structures, Structural Dynamics, and Materials Conference and Exhibit, Seattle, Washington, April 16-19 2001, AIAA Paper No. 2001-1523.

${ }^{11}$ Schimdt, D. K. and Raney, D. L., "Modeling and Simulation of Flexible Flight Vehicles," AIAA Modeling and Simulation Technologies Conference and Exhibit, Boston, Massachusetts, August 10-12 1998, AIAA Paper No. 98-4359.

${ }^{12}$ Chavez, F. R. and Schimdt, D. K., "Systems Approach to Characterizing Aircraft Aeroelastic Model Variation for Robust Control Applications," AIAA Guidance, Navigation, and Control Conference and Exhibit, Montreal, Canada, August 6-9 2001, AIAA Paper No. 2001-4020.

${ }^{13}$ van Schoor, M. C. and von Flotow, A. H., "Aeroelastic Characteristics of a Highly Flexible Aircraft," Journal of Aircraft, Vol. 27, No. 10, October 1990, pp. 901-908.

${ }^{14}$ Drela, M., "Integrated Simulation Model for Preliminary Aerodynamic, Structural, and Control-Law Design of Aircraft," 40th AIAA/ASME/ASCE/AHS/ASC Structures, Structural Dynamics, and Materials Conference and Exhibit, St. Louis, Missouri, April 12-15 1999, pp. 1644-1656, AIAA Paper No. 99-1394.

${ }^{15}$ Cesnik, C. E. S. and Brown, E. L., "Modeling of High Aspect Ratio Active Flexible Wings for Roll Control," Proceedings of the 43rd AIAA/ASME/ASCE/AHS Structures, Structural Dynamics, and Materials Conferences, Denver, Colorado, April 22-25 2002, AIAA Paper No. 2002-1719.

${ }^{16}$ Cesnik, C. E. S. and Brown, E. L., "Active Wing Warping Control of a Joined-Wing Airplane Configuration," Proceedings of the 44th AIAA/ASME/ASCE/AHS Structures, Structural Dynamics, and Materials Conferences, Norfolk, Virgina, April 7-10 2003, AIAA Paper No. 2003-1715.

${ }^{17}$ Noll, T. E., Brown, J. M., Perez-Davis, M. E., Ishmael, S. D., Tiffany, G. C., and Gaier, M., "Investigation of the Helios Prototype Aircraft Mishap," Tech. rep., NASA, January 2004. 
${ }^{18}$ Stevens, B. L. and Lewis, F. L., Aircraft Control and Simulation, John Wiley \& Sons, Inc., New York, 1992. 1983.

${ }^{19}$ Reid, J. G., Linear System Fundamentals Continuous and Discrete, Classic and Modern, McGraw-Hill, Inc., New York,

${ }^{20}$ Bay, J. S., Fundamentals of Linear State Space Systems, WCB/McGraw-Hill, Boston, 1999.

${ }^{21}$ Brown, E. L., Integrated Strain Actuation in Aircraft with Highly Flexible Composite Wings, Ph.D. thesis, Massachusetts Institute of Technology, Boston, Massachusetts, June 2003, PhD Thesis.

${ }^{22}$ Peters, D. and Johnson, M. J., "Finite-State Airloads for Deformable Airfoils on Fixed and Rotating Wings," Aeroelasticity and Fluid/Structure Interation, Proceedings of the Winter Annual Meeting, ASME, November 6-11 1994.

${ }^{23}$ Peters, D. A. and Cao, W., "Finite State Induced Flow Models Part I: Two-Dimensional Thin Airfoil," Journal of Aircraft, Vol. 32, No. 2, March-April 1995, pp. 313-322.

${ }^{24}$ Jansen, K. E., Whiting, C. H., and Hulbert, G. M., "A Generalized- $\alpha$ Method for Integrating the Filtered Navier-Stokes Equations with a Stabilized Finite Element Method," Computer Methods in Applied Mechanics and Engineering, Vol. 190, No. 3-4, 27 October 2000, pp. 305-319.

${ }^{25}$ Chung, J. and Hulbert, G. M., "A Time Integration Algorithm for Structural Dynamics With Improved Numerical Dissipation: The Generalized- $\alpha$ Method," Journal of Applied Mechanics, Vol. 60, June 1993, pp. 371-375.

${ }^{26}$ Geradin, M. and Rixen, D., Mechanical Vibrations: Theory and Applications to Structural Dynamics, John Wiley and Sons Ltd., 2nd ed., May 1997.

${ }^{27}$ Kreyszig, E., Advanced Engineering Mathematics, John Wiley \& Sons, Inc., New York, 7th ed., 1993.

\section{Appendix - Jacobian Matrices}

To reduce the total virtual work, Eq. 67, to a set of independent coordinates, the absolute variations between the dependent coordinates, $h, p$, and, $\theta$ (a rotation angle), and the independent coordinates, $\epsilon$ and $b$ are given by

$$
\begin{aligned}
d h & =J_{h \epsilon} d \epsilon+J_{h b} d b \\
d p & =J_{p \epsilon} d \epsilon+J_{p \beta} d b \\
d \theta & =J_{\theta \epsilon} d \epsilon+J_{\theta \beta} d b
\end{aligned}
$$

with the six strain-dependent Jacobians matrices, $J_{h \epsilon}, J_{h b}, J_{p \epsilon}, J_{p \beta}, J_{\theta \epsilon}$ and, $J_{\theta \beta}$, relating the variation of $h$ to $\epsilon, h$ to $b, p$ to $\epsilon$, etc. As an example, the of $J_{h \epsilon}$ is presented here for a single beam as seen in Figure 3 . The remaining Jacobinans $J_{p \epsilon}, J_{\theta \epsilon}, J_{p \beta}$ and $J_{\theta \beta}$ are not derived here but can be found in Ref. 21 .

As indicated in Eq. 46, the equations represented by the $h$ column vector were developed with $\mathbf{h}_{b c}$ as a $12 \times 1$ column vector of the boundary conditions. Taking the partials of $h_{i, j}^{1}$ with respect to $\boldsymbol{\epsilon}_{i}$, several of the terms are equal to zero, where $\boldsymbol{\epsilon}_{i}$ refers to the $i$-th element vector of strains

$$
\boldsymbol{\epsilon}_{i}=\left\{\begin{array}{llll}
\epsilon_{x_{i}} & \kappa_{x_{i}} & \kappa_{y_{i}} & \kappa_{z_{i}}
\end{array}\right\}^{T}
$$


The partial derivatives with respect to $\boldsymbol{\epsilon}_{1}$ are

$$
\begin{aligned}
& {\left[\frac{\partial}{\partial \boldsymbol{\epsilon}_{1}} h_{1,1}^{1}\right]=\left[\frac{\partial}{\partial \boldsymbol{\epsilon}_{1}} h_{b c}\right]=0} \\
& {\left[\frac{\partial}{\partial \boldsymbol{\epsilon}_{1}} h_{1,2}^{1}\right]=\left[\frac{\partial}{\partial \boldsymbol{\epsilon}_{1}} e^{\bar{G}_{1}}\right] h_{b c}} \\
& {\left[\frac{\partial}{\partial \boldsymbol{\epsilon}_{1}} h_{1,3}^{1}\right]=\left[\frac{\partial}{\partial \boldsymbol{\epsilon}_{1}} e^{2 \bar{G}_{1}}\right] h_{b c}} \\
& {\left[\frac{\partial}{\partial \boldsymbol{\epsilon}_{1}} h_{2,1}^{1}\right]=D_{2,1}^{1}\left[\frac{\partial}{\partial \boldsymbol{\epsilon}_{1}} e^{2 \bar{G}_{1}}\right] h_{b c}} \\
& {\left[\frac{\partial}{\partial \boldsymbol{\epsilon}_{1}} h_{2,2}^{1}\right]=e^{\bar{G}_{2}} D_{2,1}^{1}\left[\frac{\partial}{\partial \boldsymbol{\epsilon}_{1}} e^{2 \bar{G}_{1}}\right] h_{b c}} \\
& {\left[\frac{\partial}{\partial \boldsymbol{\epsilon}_{1}} h_{2,3}^{1}\right]=e^{2 \bar{G}_{2}} D_{2,1}^{1}\left[\frac{\partial}{\partial \boldsymbol{\epsilon}_{1}} e^{2 \bar{G}_{1}}\right] h_{b c}} \\
& {\left[\frac{\partial}{\partial \boldsymbol{\epsilon}_{1}} h_{3,1}^{1}\right]=D_{3,2}^{1} e^{2 \bar{G}_{2}} D_{2,1}^{1}\left[\frac{\partial}{\partial \boldsymbol{\epsilon}_{1}} e^{2 \bar{G}_{1}}\right] h_{b c}} \\
& {\left[\frac{\partial}{\partial \boldsymbol{\epsilon}_{1}} h_{3,2}^{1}\right]=e^{\bar{G}_{3}} D_{3,2}^{1} e^{2 \bar{G}_{2}} D_{2,1}^{1}\left[\frac{\partial}{\partial \boldsymbol{\epsilon}_{1}} e^{2 \bar{G}_{1}}\right] h_{b c}}
\end{aligned}
$$

and with respect to $\boldsymbol{\epsilon}_{2}$

$$
\begin{aligned}
& {\left[\frac{\partial}{\partial \boldsymbol{\epsilon}_{2}} h_{1,1}^{1}\right]=0} \\
& {\left[\frac{\partial}{\partial \boldsymbol{\epsilon}_{2}} h_{1,2}^{1}\right]=0} \\
& {\left[\frac{\partial}{\partial \boldsymbol{\epsilon}_{2}} h_{1,3}^{1}\right]=0} \\
& {\left[\frac{\partial}{\partial \boldsymbol{\epsilon}_{2}} h_{2,1}^{1}\right]=0} \\
& {\left[\frac{\partial}{\partial \boldsymbol{\epsilon}_{2}} h_{2,2}^{1}\right]=\left[\frac{\partial}{\partial \boldsymbol{\epsilon}_{2}} e^{\bar{G}_{2}}\right] D_{2,1}^{1} e^{2 \bar{G}_{1}} h_{b c}} \\
& {\left[\frac{\partial}{\partial \boldsymbol{\epsilon}_{2}} h_{2,3}^{1}\right]=\left[\frac{\partial}{\partial \boldsymbol{\epsilon}_{2}} e^{2 \bar{G}_{2}}\right] D_{2,1}^{1} e^{2 \bar{G}_{1}} h_{b c}} \\
& {\left[\frac{\partial}{\partial \boldsymbol{\epsilon}_{2}} h_{3,1}^{1}\right]=D_{3,2}^{1}\left[\frac{\partial}{\partial \boldsymbol{\epsilon}_{2}} e^{2 \bar{G}_{2}}\right] D_{2,1}^{1} e^{2 \bar{G}_{1}} h_{b c}} \\
& {\left[\frac{\partial}{\partial \boldsymbol{\epsilon}_{2}} h_{3,2}^{1}\right]=e^{\bar{G}_{3}} D_{3,2}^{1}\left[\frac{\partial}{\partial \boldsymbol{\epsilon}_{2}} e^{2 \bar{G}_{2}}\right] D_{2,1}^{1} e^{2 \bar{G}_{1}} h_{b c}}
\end{aligned}
$$


Along a single beam the following recursive relationship is found

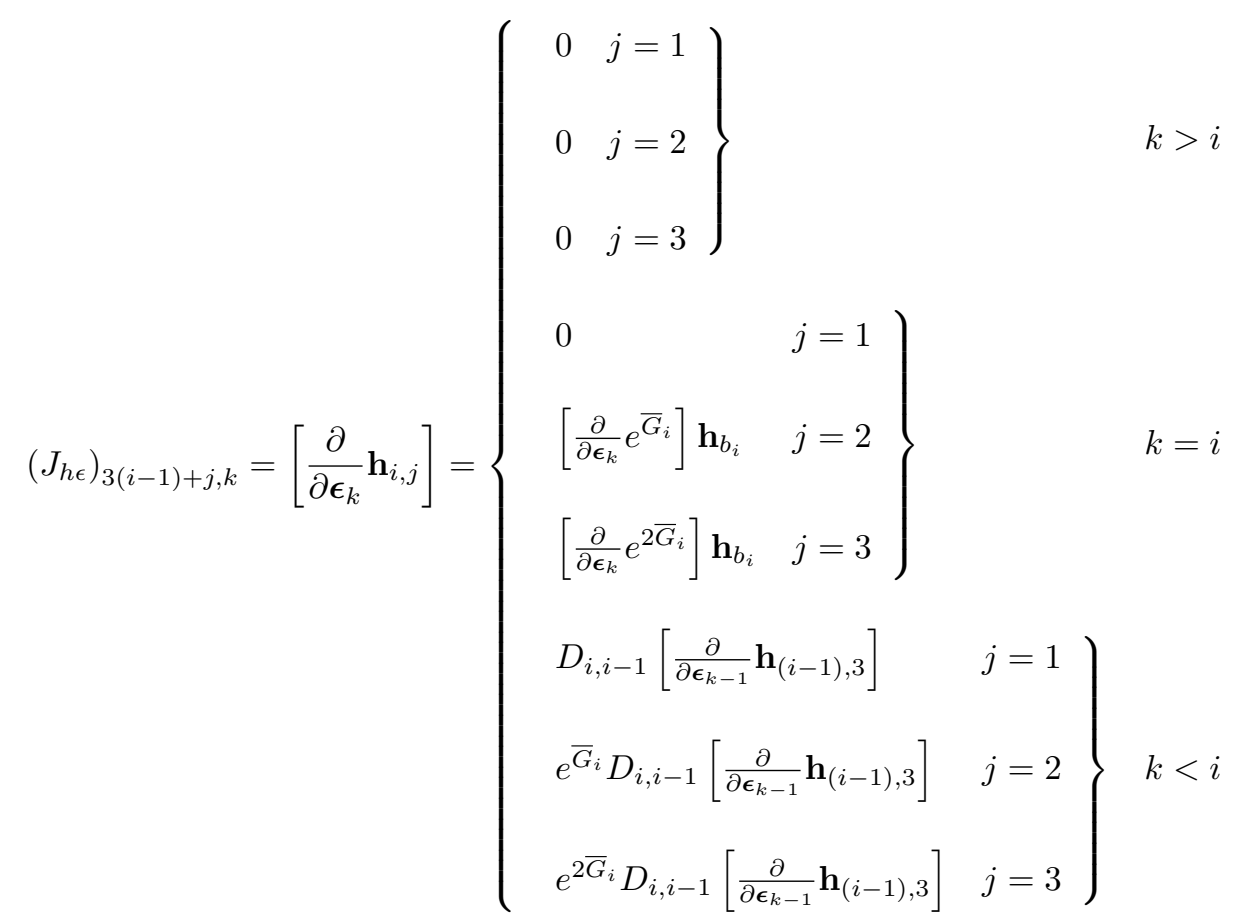

where $i, k$ represent element numbers and $j$ represents nodal numbers, with $j=(1,2,3)$. The boundary condition $\mathbf{h}_{b_{i}}$ is

$$
\mathbf{h}_{b_{i}}=\left\{\begin{array}{cc}
h_{b c} & i=0 \\
D_{i, i-1} \mathbf{h}_{i-1,3} & i>0
\end{array}\right.
$$

Finally $J_{h \epsilon}$ is written as

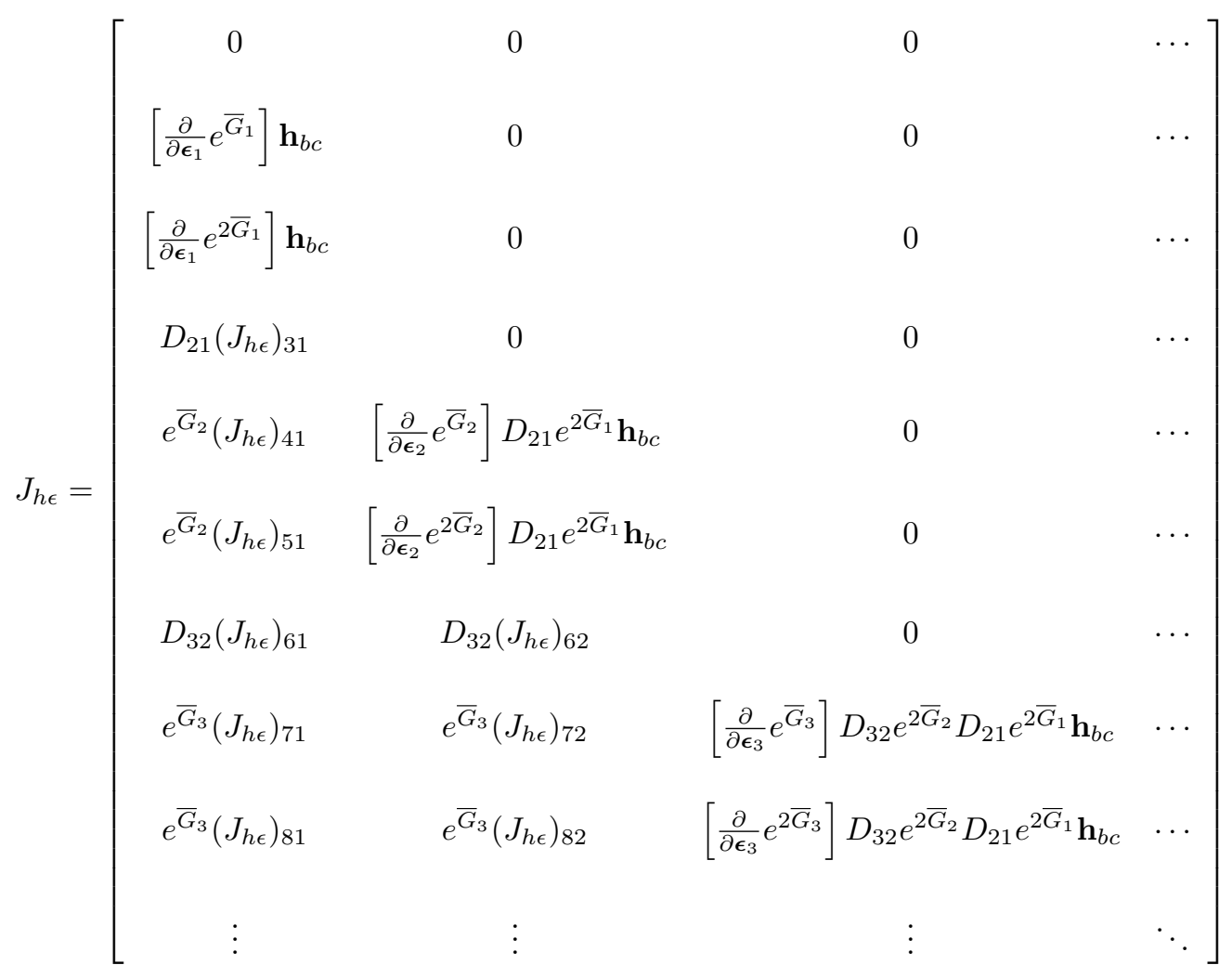


Note that each entry of $J_{h \epsilon}$ is a $12 \times 4$ matrix. 